\title{
Moving Target Imaging and Ghost Mitigation in Through-the-Wall Sensing Application
}

\author{
Xin Sun, BiYing Lu, and ZhiMin Zhou \\ College of Electronic Science and Engineering, National University of Defense Technology, Changsha, Hunan 410073, China \\ Correspondence should be addressed to Xin Sun; jaddymail@163.com
}

Received 29 October 2013; Revised 25 February 2014; Accepted 13 March 2014; Published 2 July 2014

Academic Editor: Lorenzo Crocco

Copyright (C) 2014 Xin Sun et al. This is an open access article distributed under the Creative Commons Attribution License, which permits unrestricted use, distribution, and reproduction in any medium, provided the original work is properly cited.

\begin{abstract}
Human is one kind of the most interesting targets in through-the-wall imaging. In high-resolution imaging applications, human is no longer a point target. Therefore, the previous signal models constructed by point target assumption cannot accurately describe real characteristics of EM propagation. We construct the signal models based on extended target theory in this paper. Compared with previous works, the main contributions are as follows. Firstly, human is considered as an extended target. The expressions of target scattering and wall reflections are derived. Secondly, target scattering is no longer isotropic in new model. A new kind of ghost problem which is caused by target obscuring in EM propagation is discovered and exploited. Thirdly, to improve image quality in moving target imaging, an efficient approach which adopts CFAR, clustering method, and spatial geometry relationship is proposed to remove the ghosts. The derived models are shown to agree with synthetic and experimental results. And the efficiency of proposed method is also validated, which illuminates that the ghosts are efficiently mitigated and the image quality is significantly improved.
\end{abstract}

\section{Introduction}

Ultra-wideband (UWB) through-wall-imaging (TWI) approaches that can detect objects through obstacles, such as walls, doors, and other opaque materials, are considered as a powerful tool for a variety of civilian and military applications [1-5]. In real TWI application, human is one kind of the most interesting targets [6-10]. Studying human imaging, especially moving human imaging, is quite significant. Therefore, the corresponding moving target imaging (MTI) technique is widely researched in TWI application [11-15].

For exploiting the MTI, an accurate signal model is necessary and urgently required. Many efforts have been made to analyze the signal models to improve image quality. In $[16,17]$, an inverse scattering signal model is derived. Based on the model, tomography imaging method and change detection technique are adopted in MTI processing. Besides inverse scattering signal model, a back projection signal model is discussed in [18-20]. Electromagnetic synthetic experiments are given in this paper. In order to analyze the EM propagation in a building, ray multipath is computed with ATrace for a building structure, and all paths linking a transmitter-receiver are discovered in [21]. And in [22, 23], a multipath model for sensing through walls is proposed. To sum up, it is noted that all these models consider the target as point one and assume its scattering to be isotropic. Since the target is point one, effects of the target itself on EM propagation are not paid enough attention in these models.

However, in high-resolution imaging radar applications [24-27], most of the targets, such as human and furniture, are no longer point targets. For example, in a common highresolution through-the-wall radar system, the frequency range is usually $1-3 \mathrm{GHz}$ or even larger. If the frequency band is $2 \mathrm{GHz}$, the range resolution is $7.5 \mathrm{~cm}$. However, the size of a common human is $1.8 \mathrm{~m} \times 0.5 \mathrm{~m} \times 0.35 \mathrm{~m}$ [28, 29]. In this case, the human will occupy several resolution units. Considering the relationship between human size and resolution, human must be considered as an extended target. So, in order to describe the echo and analyze its effects more accurately, the signal models need to be reconstructed by extended target theory. 


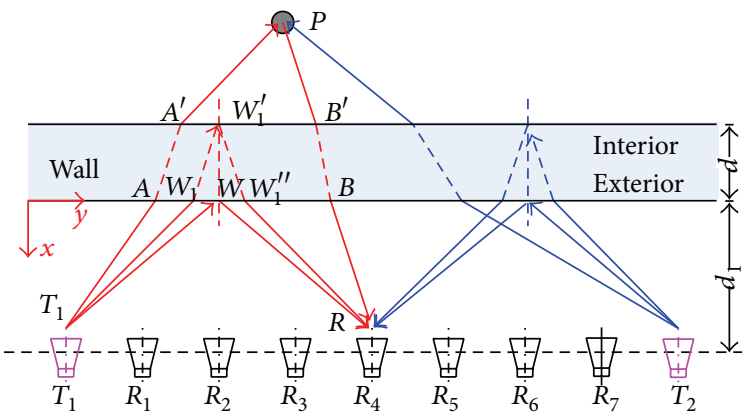

(a)

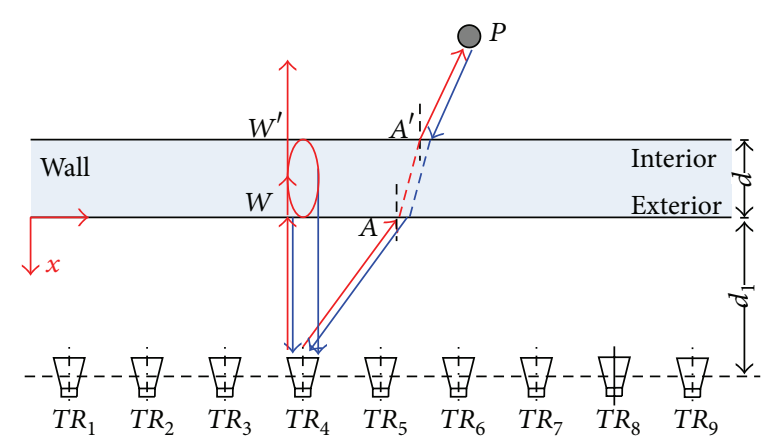

(b)

FIGURE 1: The conventional TWI point target signal models: (a) MIMO mode; (b) monostatic mode.

In this paper, we construct the TWI signal models using the extended target theory in two typical applications: the MIMO through-the-wall mode and monostatic through-thewall mode. Based on the models, a new kind of ghost is discovered and exploited. To improve the image quality, an efficient ghost mitigation approach is proposed. The paper is organized as follows. In Section 2, compared with the conventional point target signal models, two new signal models, for MIMO and monostatic modes, are constructed by extended target theory. Then moving target imaging for extended target is discussed in Section 3. The ghost caused by target obscuring is discovered and analyzed. In Section 4 , to improve the imaging quality, a ghost mitigation method which applies CFAR, clustering method, and spatial geometry relationship is proposed. Simulations and experiments are given in Section 5. Conclusions end this paper.

\section{TWI Signal Model}

After comparing with the conventional point target signal models, two new signal models for extended target, in MIMO and monostatic modes, are constructed in this section.

2.1. Conventional Point Target Signal Model. In TWI applications, transmissivity coefficient and the reflectivity coefficient can be measured or computed by EM models. Besides, building walls, such as brick, adobe, and poured concrete walls, can be modeled by homogeneous dielectric slabs. Its transmission coefficient, $\Gamma$, and the reflection coefficient, $\gamma$, are given in [30]. Their expressions are

$$
\begin{gathered}
\Gamma=\frac{4 e^{j\left(k_{1 x}-k_{0 x}\right) d}}{\left(1+p_{01}\right)\left(1+p_{10}\right)\left(1+R_{01} R_{10} e^{2 i k_{1 x} d}\right)}, \\
\gamma=\frac{R_{01}+R_{10} e^{i 2 k_{1 x} d}}{\left(1+R_{01} R_{10} e^{i 2 k_{1 x} d}\right)},
\end{gathered}
$$

where $d$ is the thickness of the wall and $k_{0 x}=2 \pi f_{m} \cos \varphi_{i} / c$ and $k_{1 x}=2 \pi \sqrt{\varepsilon} f_{m} \cos \varphi_{r} / c$ are the normal components of the propagation constants in the air and in the wall, respectively. Here $f_{m}$ is the $m$ th frequency point. $c$ is the velocity of EM in free space, and $\varepsilon$ is the relative permittivity of the wall. $\varphi_{i}$ and $\varphi_{r}$ are the incident angle and refraction angle in the air-wall interface. $p_{10}$ is the ratio of $k_{0 x}$ to $k_{1 x}$; $p_{10}=k_{0 x} / k_{1 x}$ for horizontal polarization, and $p_{10}=\varepsilon k_{0 x} / k_{1 x}$ for vertical polarization. $p_{01}$ is the reciprocal of $p_{10}$; that is, $p_{01}=1 / p_{10}$. The expressions of variables $R_{10}$ and $R_{01}$ are

$$
R_{10}=\frac{1-p_{10}}{1+p_{10}}, \quad R_{01}=\frac{p_{10}-1}{1+p_{10}} .
$$

Using the Taylor series method, the expanded form of (2) is

$$
\gamma=-R_{10}+\frac{\left[1-\left(R_{10}\right)^{2}\right]}{R_{10}} \cdot \sum_{n=1}^{\infty}\left(R_{10}\right)^{2 n} \exp \left[j 2 n k_{1 x} \cdot d\right],
$$

where $n$ denotes the reflection times when EM wave propagates inside the wall. The first term of (4) is the reflection coefficient of wall exterior. The second term corresponds to the reflection coefficient of the first reflection from the interior and multireflection inside the wall.

When EM wave propagates in the wall, the energy of multireflections is rather weak because of amplitude attenuation. Therefore, wall echo is mainly composed of the first reflections from the exterior and interior. For convenience hereinafter, the multi-reflections are neglected in the following derivation. After considering the penetration losses in the wall, the reflections coefficients for the exterior and interior can be obtained from (5). One has

$$
\begin{gathered}
\Lambda_{\text {exterior }}=\left|R_{10}\right|, \\
\Lambda_{\text {interior }}=\alpha\left|\left[R_{10}-\left(R_{10}\right)^{3}\right] \exp \left(j 2 k_{1 x} \cdot d\right)\right| .
\end{gathered}
$$

Here, the variable $\alpha$ denote EM penetration losses of the interior reflection.

(A) MIMO Through-the-Wall Mode. Figure 1(a) is the conventional point target signal model in the MIMO mode. In the figure, we take an antenna array with two transmitters and seven receivers as an example. EM illuminated by $T_{1}$ is marked with red lines and that transmitted by $T_{2}$ is marked with blue lines. 
Therefore, taking stepped frequency signal, for example, the wall echo received by antenna $R$ is the sum of the returns corresponding to all the transmitters. One has

$$
\begin{aligned}
s_{w}^{f}\left(t, \mathbf{x}_{R}\right) & \\
=\mathrm{FT}_{t \leftrightarrow k}^{-1}\left\{\sum_{i=1}^{2}[\right. & A_{w} \cdot Z_{i}\left(k_{m}\right) \cdot \Lambda_{\text {exterior }}\left(\mathbf{x}_{T_{i}}, \mathbf{x}_{R}, k_{m}\right) \\
& \cdot \exp \left(-j k_{m} \Delta l_{1}^{f}\left(\mathbf{x}_{T_{i}}, \mathbf{x}_{R}\right)\right) \\
& +A_{w} \cdot Z_{i}\left(k_{m}\right) \cdot \Lambda_{\text {interior }}\left(\mathbf{x}_{T_{i}}, \mathbf{x}_{R}, k_{m}\right) \\
& \left.\left.\cdot \exp \left(-j k_{m} \Delta l_{2}^{f}\left(\mathbf{x}_{T_{i}}, \mathbf{x}_{R}\right)\right)\right]\right\},
\end{aligned}
$$

where $s_{w}^{f}\left(t, \mathbf{x}_{R}\right)$ denotes the wall echo received by receiver $R . A_{w}$ is the total factor involving EM wave propagation attenuation and the influence of the antenna footprint. $k_{m}$ is the wave number with $k_{m}=2 \pi f_{m} / c . \mathrm{FT}_{t \leftrightarrow k}^{-1}(\cdot)$ is the inverse Fourier transform operator with respect to $t . Z_{i}(\cdot)$ is the spectrum of the signal transmitted by antenna $T_{i} \cdot \mathbf{x}_{T_{i}}$ and $\mathbf{x}_{R}$ are the positions of transmitter $T_{i}$ and receiver $R$, with $\mathbf{x}_{T_{i}}=\left(x_{T_{i}}, y_{T_{i}}\right)$ and $\mathbf{x}_{R}=\left(x_{R}, y_{R}\right)$, respectively. The variables $\Delta l_{1}^{f}(\cdot)$ and $\Delta l_{2}^{f}(\cdot)$ are signal distance of the exterior reflection and interior reflection, respectively. Taking $T_{1}$ in Figure 1(a), for example, the expressions of $\Delta l_{1}^{f}(\cdot)$ and $\Delta l_{2}^{f}(\cdot)$ are

$$
\begin{gathered}
\Delta l_{1}^{f}\left(\mathbf{x}_{T_{1}}, \mathbf{x}_{R}\right)=\left\|\mathbf{x}_{T_{1}} \mathbf{x}_{W}\right\|+\left\|\mathbf{x}_{W} \mathbf{x}_{R}\right\|, \\
\Delta l_{2}^{f}\left(\mathbf{x}_{T_{1}}, \mathbf{x}_{R}\right) \\
=\left\|\mathbf{x}_{T_{1}} \mathbf{x}_{W_{1}}\right\|+\left(\left\|\mathbf{x}_{W_{1}} \mathbf{x}_{W_{1}^{\prime}}\right\|+\left\|\mathbf{x}_{W_{1}^{\prime}} \mathbf{x}_{W_{1}^{\prime \prime}}\right\|\right) \cdot \sqrt{\varepsilon}+\left\|\mathbf{x}_{W_{1}^{\prime \prime}} \mathbf{x}_{R}\right\| .
\end{gathered}
$$

In (7a), $\mathbf{x}_{W}$ is a reflection position on air-wall interface. In (7b), $\mathbf{x}_{W_{1}}$ and $\mathbf{x}_{W_{1}^{\prime \prime}}$ are refraction positions on air-wall interface and wall-air interface, respectively. $\mathbf{x}_{W_{1}^{\prime}}$ is the reflection position on wall-air interface of the wall. The operation $\|\cdot\|$ denotes the Descartes range.

Based on the model, returns of the point target scattering are

$$
\begin{aligned}
s_{g}\left(t, \mathbf{x}_{R}\right)=\mathrm{FT}_{t \leftrightarrow k}^{-1}\left\{\sum_{i=1}^{2}[\right. & A_{g} \cdot Z_{i}\left(k_{m}\right) \cdot \Gamma\left(\mathbf{x}_{T_{i}}, \mathbf{x}_{P}, k_{m}\right) \\
\cdot & \Gamma\left(\mathbf{x}_{P}, \mathbf{x}_{R}, k_{m}\right) \\
\cdot & \left.\left.\exp \left(-j k_{m} \Delta l_{g}\left(\mathbf{x}_{T_{i}}, \mathbf{x}_{P}, \mathbf{x}_{R}\right)\right)\right]\right\},
\end{aligned}
$$

where $s_{g}\left(t, \mathbf{x}_{R}\right)$ is the target echo received by antenna $R$. $A_{g}$ is the factor involving target scattering amplitude, EM wave propagation attenuation, and the influence of the antenna footprint. $\Delta l_{g}(\cdot)$ is the signal distance of target scattering.
Taking $T_{1}$ in Figure 1(a), for example, the expression of $\Delta l_{g}(\cdot)$ is

$$
\begin{aligned}
\Delta l_{g}\left(\mathbf{x}_{T_{1}}, \mathbf{x}_{P}, \mathbf{x}_{R}\right)= & \left\|\mathbf{x}_{T_{1}} \mathbf{x}_{A}\right\|+\left\|\mathbf{x}_{A} \mathbf{x}_{A^{\prime}}\right\| \cdot \sqrt{\varepsilon}+\left\|\mathbf{x}_{A^{\prime}} x_{P}\right\| \\
& +\left\|\mathbf{x}_{P} \mathbf{x}_{B^{\prime}}\right\|+\left\|\mathbf{x}_{B^{\prime}} \mathbf{x}_{B}\right\| \cdot \sqrt{\varepsilon}+\left\|\mathbf{x}_{B} \mathbf{x}_{R}\right\|,
\end{aligned}
$$

where $\mathbf{x}_{A}$ and $\mathbf{x}_{B}$ are refraction positions on air-wall interface and wall-air interface of the exterior, respectively, and $\mathbf{x}_{A^{\prime}}$ and $\mathbf{x}_{B^{\prime}}$ are refraction positions on wall-air interface and air-wall interface of the interior, respectively.

(B) Monostatic Through-the-Wall Mode. Figure 1(b) shows the conventional point target signal model for monostatic through-wall-imaging applications. In this mode, signal is emitted and received by the same antenna. The antennas work alternately. Based on the signal model, wall reflection is computed by

$$
\begin{aligned}
s_{w}^{f}\left(t, \mathbf{x}_{T R}\right)=\mathrm{FT}_{t \leftrightarrow k}^{-1}\left\{A_{w} \cdot Z\left(k_{m}\right) \cdot \Lambda_{\text {exterior }}\left(\mathbf{x}_{T R}, k_{m}\right)\right. \\
\cdot \exp \left(-j k_{m} \Delta l_{1}^{f}\left(\mathbf{x}_{T R}\right)\right) \\
+A_{w} \cdot Z\left(k_{m}\right) \cdot \Lambda_{\text {interior }}\left(\mathbf{x}_{T R}, k_{m}\right) \\
\left.\cdot \exp \left(-j k_{m} \Delta l_{2}^{f}\left(\mathbf{x}_{T R}\right)\right)\right\} .
\end{aligned}
$$

Here, the signal distances of the wall exterior and interior, that is, $\Delta l_{1}^{f}(\cdot)$ and $\Delta l_{1}^{f}(\cdot)$, are

$$
\begin{gathered}
\Delta l_{1}^{f}(\cdot)=2 d_{1}, \\
\Delta l_{2}^{f}(\cdot)=2\left(d_{1}+d \cdot \sqrt{\varepsilon}\right),
\end{gathered}
$$

where $d_{1}$ is the distance from the antenna to the wall.

Anonymously, the point target scattering is

$$
\begin{array}{r}
s_{g}\left(t, \mathbf{x}_{T R}\right)=\mathrm{FT}_{t \leftrightarrow k}^{-1}\left\{A_{g} \cdot Z\left(k_{m}\right) \cdot \Gamma^{2}\left(\mathbf{x}_{T R}, \mathbf{x}_{P}, k_{m}\right)\right. \\
\left.\cdot \exp \left(-j k_{m} \Delta l_{g}\left(\mathbf{x}_{T R}, \mathbf{x}_{P}\right)\right)\right\},
\end{array}
$$

where the signal distance of target scattering is

$$
\Delta l_{g}\left(\mathbf{x}_{T R}, \mathbf{x}_{P}\right)=2\left(\left\|\mathbf{x}_{T R} \mathbf{x}_{A}\right\|+\left\|\mathbf{x}_{A_{A}} \mathbf{x}_{A^{\prime}}\right\| \cdot \sqrt{\varepsilon}+\left\|\mathbf{x}_{A^{\prime}} \mathbf{x}_{P}\right\|\right) .
$$

2.2. TWI Signal Model for Extended Target. As mentioned above, target is considered as an isotropic point target in conventional models. However, in real TWI applications, most of the targets, such as the human and furniture, are extended targets rather than point targets. And these scatterings are no longer isotropic. Therefore, the signal models for TWI applications need to be reconstructed.

In the new models, we assume a simple scene where a human is placed in a four-wall room (Figure 2). The human is located at $\mathbf{x}_{p}=\left(x_{p}, y_{p}\right)$. Considering amplitude attenuation, reflections from the interior on the back wall and the multipath components are neglected in the derivation.

(A) MIMO Through-the-Wall Mode. In the new signal model, reflection from the front wall is the same as that in point target 


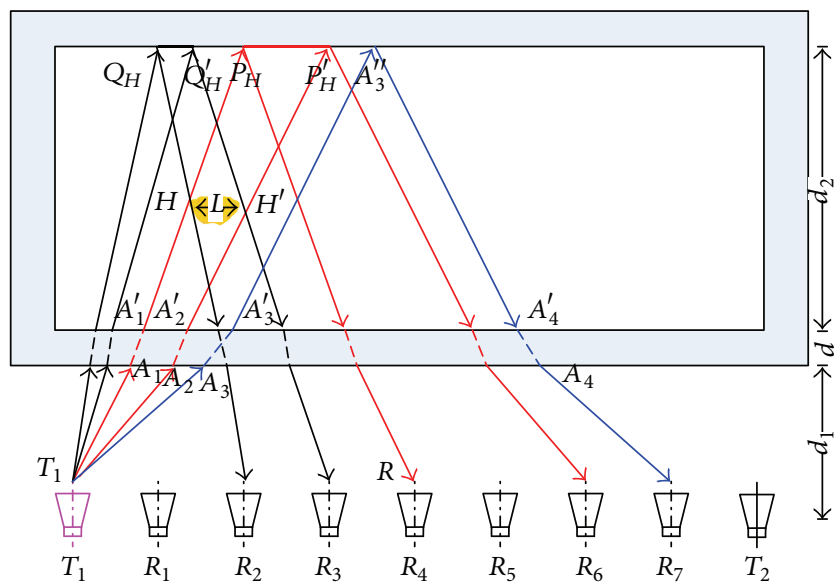

(a)

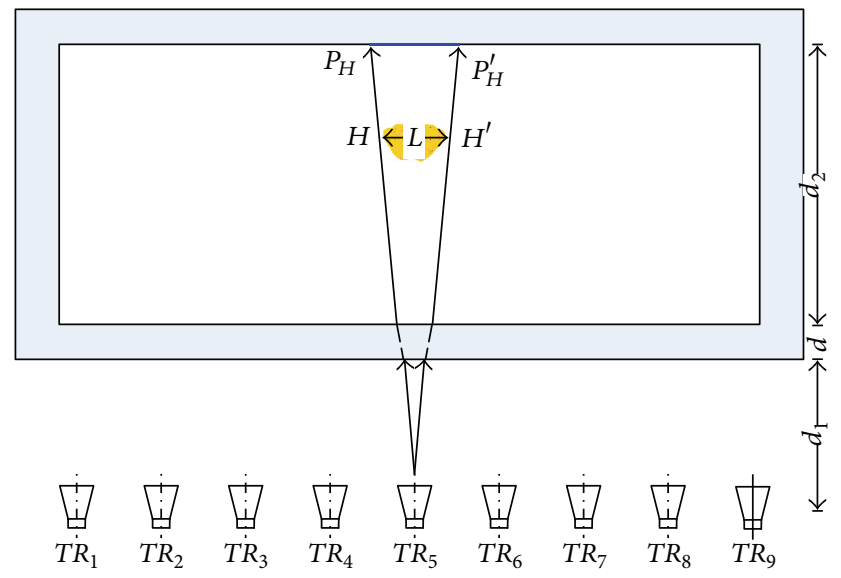

(b)

FIGURE 2: Reflections from the back wall in extended target signal model for the MIMO and monostatic TWI applications. (a) The MIMO mode. In this figure, the antenna array includes two transmitters and seven receivers. For convenience, only the signal channels transmitted by $T_{1}$ are marked. (b) The monostatic mode. In this figure, EM waves emitted by $T R_{5}$ are mainly obscured by the target.

signal model, that is, (6). But the target scattering is changed. As an extended one, the target scattering is consequently the sum of the returns from its scattering points. Therefore, the extended target returns received by antenna $\mathbf{x}_{R}$ can be expressed as

$$
\begin{aligned}
& s_{g}\left(t, \mathbf{x}_{R}\right) \\
&=\mathrm{FT}_{t \leftrightarrow k}^{-1}\left\{\sum_{n=1}^{N} \sum_{i=1}^{2}[\right. A_{g}\left(\mathbf{x}_{U_{n}}\right) \cdot Z_{i}\left(k_{m}\right) \cdot \Gamma\left(\mathbf{x}_{T_{i}}, \mathbf{x}_{U_{n}}, k_{m}\right) \\
& \cdot \Gamma\left(\mathbf{x}_{U_{n}}, \mathbf{x}_{R}, k_{m}\right) \\
&\left.\left.\cdot \exp \left(-j k_{m} \Delta l_{g}\left(\mathbf{x}_{T_{i}}, \mathbf{x}_{U_{n}}, \mathbf{x}_{R}\right)\right)\right]\right\},
\end{aligned}
$$

where $\mathbf{x}_{U_{n}}$ denotes the $n$th scattering point of the target. $N$ is the count of scatter points. $A_{g}(\cdot)$ is the factor of each scattering point.

Because the target is an extended one, it will partially obscure the EM waves. In Figure 2, $H$ and $H^{\prime}$ denote the left end and right ends of the target. When EM propagates through the front wall, the waves get partially reflected by the back wall and partially obscured by the target (shown in Figure 2(a)). Now, we analyze the range of the obscured area on the back wall.

Taking antenna $T_{1}$, for example, when EM is illuminated by transmitter $T_{1}$, there are two cases about obscuring (shown in Figure 2(a)). One occurs before the reflection from the back wall (marked in red lines); the other occurs after the reflection from the back wall (marked in black lines). In the first case, the obscured area, $\Pi_{11}=\left(x_{\mathrm{ob}}, y_{\mathrm{ob}}\right)$, on the back wall conforms to (15a), (15b), and (15c). Here, variable $L$ denotes the length of the target along the cross range axis,

$$
\begin{aligned}
\Pi_{11}= & \left\{\begin{array}{l}
x_{\mathrm{ob}} \in\left[\begin{array}{ll}
x_{P_{H}} & x_{P_{H}^{\prime}}
\end{array}\right] \\
y_{\mathrm{ob}}=d_{1}+d+d_{2}+y_{T_{1}}
\end{array} x_{p}>x_{T_{1}}+\frac{L}{2},\right. \\
x_{P_{H}}= & x_{p}-\frac{L}{2} \\
& +\left(\left(x_{p}-\frac{L}{2}-x_{T_{1}}\right)\left(\sqrt{\varepsilon} y_{p}-\sqrt{\varepsilon} y_{T_{1}}-\sqrt{\varepsilon} d-d\right)\right. \\
& \left.\quad \times\left(y_{T_{1}}+d_{1}+d+d_{2}-y_{p}\right)\right) \\
& \times\left(\sqrt{\varepsilon}\left(y_{p}-y_{T_{1}}-d\right)\left(y_{p}-y_{T_{1}}-d_{1}\right)-d d_{1}\right)^{-1}, \\
& \quad x_{p}>x_{T_{1}}+\frac{L}{2},
\end{aligned}
$$

$$
\begin{aligned}
x_{P_{H}^{\prime}}= & x_{p}+\frac{L}{2} \\
+ & \left(\left(x_{p}+\frac{L}{2}-x_{T_{1}}\right)\left(\sqrt{\varepsilon} y_{p}-\sqrt{\varepsilon} y_{T_{1}}-\sqrt{\varepsilon} d-d\right)\right. \\
\left.\quad \times\left(y_{T_{1}}+d_{1}+d+d_{2}-y_{p}\right)\right) & \\
\times & \left(\sqrt{\varepsilon}\left(y_{p}-y_{T_{1}}-d\right)\left(y_{p}-y_{T_{1}}-d_{1}\right)-d d_{1}\right)^{-1}, \\
& x_{p}>x_{T_{1}}+\frac{L}{2} .
\end{aligned}
$$


In the second case, the obscured area, $\Pi_{12}$, on the back wall conforms to (16a), (16b), and (16c). The derivations about (15a), (15b), and (15c) and (16a), (16b), and (16c) are given in the Appendix. One has

$$
\begin{aligned}
& \Pi_{12}=\left\{\begin{array}{l}
x_{\mathrm{ob}} \in\left[\begin{array}{ll}
x_{\mathrm{Q}_{H}} & x_{\mathrm{Q}_{H}^{\prime}}
\end{array}\right] \\
y_{\mathrm{ob}}=d_{1}+d+d_{2}+y_{T_{1}}
\end{array} \quad x_{p}>x_{T_{1}}+\frac{L}{2},\right. \\
& x_{\mathrm{Q}_{H}}=\frac{\left(y_{p}-d_{1}-d-d_{2}\right) x_{T_{1}}-d_{2}\left(x_{p}-L / 2\right)}{y_{p}-d_{1}-d-2 d_{2}} \\
& +\left(\left(x_{p}-\frac{L}{2}-x_{T_{1}}\right) \cdot\left(y_{p}-d_{1}-d-d_{2}\right)\right. \\
& \cdot\left[\left(d_{1}^{2}+2 d d_{1}+2 d_{2} d_{1}\right) \sqrt{\varepsilon}+d^{2}\right. \\
& \left.\left.+2 d_{2} d-\left(d_{1} \sqrt{\varepsilon}+d\right) y_{p}\right]\right) \\
& \times\left(\left[\sqrt{\varepsilon}\left(2 d_{1}+d+2 d_{2}-y_{p}\right)\right.\right. \\
& \left.\times\left(d_{1}+2 d+2 d_{2}-y_{p}\right)-d d_{1}\right] \\
& \left.\times\left(y_{p}-d_{1}-d-2 d_{2}\right)\right)^{-1} \text {, } \\
& x_{p}>x_{T_{1}}+\frac{L}{2}, \\
& x_{Q_{H}^{\prime}}=\frac{\left(y_{p}-d_{1}-d-d_{2}\right) x_{T_{1}}-d_{2}\left(x_{p}+L / 2\right)}{y_{p}-d_{1}-d-2 d_{2}} \\
& +\left(\left(x_{p}+\frac{L}{2}-x_{T_{1}}\right) \cdot\left(y_{p}-d_{1}-d-d_{2}\right)\right. \\
& \cdot\left[\left(d_{1}^{2}+2 d d_{1}+2 d_{2} d_{1}\right) \sqrt{\varepsilon}\right. \\
& \left.\left.+d^{2}+2 d_{2} d-\left(d_{1} \sqrt{\varepsilon}+d\right) y_{p}\right]\right) \\
& \times\left(\left[\sqrt{\varepsilon}\left(2 d_{1}+d+2 d_{2}-y_{p}\right)\right.\right. \\
& \left.\times\left(d_{1}+2 d+2 d_{2}-y_{p}\right)-d d_{1}\right] \\
& \left.\times\left(y_{p}-d_{1}-d-2 d_{2}\right)\right)^{-1} \text {, } \\
& x_{p}>x_{T_{1}}+\frac{L}{2} \text {. }
\end{aligned}
$$

Anonymously, when EM is illuminated by transmitter $T_{2}$, there are also two cases about obscuring. In the first case, the obscured area, $\Pi_{21}$, on the back wall conforms to

$$
\Pi_{21}=\left\{\begin{array}{l}
x_{\mathrm{ob}} \in\left[\begin{array}{ll}
x_{P_{H}}^{\prime} & x_{P_{H}^{\prime}}^{\prime}
\end{array}\right] \\
y_{\mathrm{ob}}=d_{1}+d+d_{2}+y_{T_{1}}
\end{array} \quad x_{p}<x_{T_{2}}-\frac{L}{2},\right.
$$

$$
\begin{aligned}
x_{P_{H}}^{\prime}= & x_{p}-\frac{L}{2} \\
- & \left(\left(x_{T_{2}}-x_{p}+\frac{L}{2}\right)\left(\sqrt{\varepsilon} y_{p}-\sqrt{\varepsilon} y_{T_{2}}-\sqrt{\varepsilon} d-d\right)\right. \\
& \left.\times\left(y_{T_{2}}+d_{1}+d+d_{2}-y_{p}\right)\right) \\
& \times\left(\sqrt{\varepsilon}\left(y_{p}-y_{T_{2}}-d\right)\left(y_{p}-y_{T_{2}}-d_{1}\right)-d d_{1}\right)^{-1}, \\
x_{P_{H}}^{\prime}= & x_{p}+\frac{L}{2} \quad x_{T_{2}}-\frac{L}{2}, \\
- & \left(\left(x_{T_{2}}-x_{p}-\frac{L}{2}\right)\left(\sqrt{\varepsilon} y_{p}-\sqrt{\varepsilon} y_{T_{2}}-\sqrt{\varepsilon} d-d\right)\right. \\
& \left.\times\left(y_{T_{2}}+d_{1}+d+d_{2}-y_{p}\right)\right) \\
\times & \left(\sqrt{\varepsilon}\left(y_{p}-y_{T_{2}}-d\right)\left(y_{p}-y_{T_{2}}-d_{1}\right)-d d_{1}\right)^{-1}, \\
& \quad x_{p}<x_{T_{2}}-\frac{L}{2} .
\end{aligned}
$$

In the second case, the obscured area, $\Pi_{22}$, on the back wall conforms to

$$
\begin{aligned}
& \Pi_{22}=\left\{\begin{array}{l}
x_{\mathrm{ob}} \in\left[\begin{array}{ll}
x_{\mathrm{Q}_{H}}^{\prime} & x_{\mathrm{Q}_{H}^{\prime}}^{\prime}
\end{array}\right], \\
y_{\mathrm{ob}}=d_{1}+d+d_{2}+y_{T_{1}},
\end{array}\right. \\
& x_{\mathrm{Q}_{H}}^{\prime}=\frac{\left(y_{p}-d_{1}-d-d_{2}\right) x_{T_{2}}-d_{2}\left(x_{p}-L / 2\right)}{y_{p}-d_{1}-d-2 d_{2}} \\
& +\left(\left(x_{T_{2}}-x_{p}+\frac{L}{2}\right) \cdot\left(y_{p}-d_{1}-d-d_{2}\right)\right. \\
& \cdot\left[\left(d_{1}^{2}+2 d d_{1}+2 d_{2} d_{1}\right) \sqrt{\varepsilon}+d^{2}\right. \\
& \left.\left.+2 d_{2} d-\left(d_{1} \sqrt{\varepsilon}+d\right) y_{p}\right]\right) \\
& \times\left(\left[\sqrt{\varepsilon}\left(2 d_{1}+d+2 d_{2}-y_{p}\right)\right.\right. \\
& \left.\times\left(d_{1}+2 d+2 d_{2}-y_{p}\right)-d d_{1}\right] \\
& \left.\times\left(y_{p}-d_{1}-d-2 d_{2}\right)\right)^{-1}, \\
& x_{p}<x_{T_{2}}-\frac{L}{2},
\end{aligned}
$$




$$
\begin{aligned}
x_{Q_{H}^{\prime}}^{\prime}= & \frac{\left(y_{p}-d_{1}-d-d_{2}\right) x_{T_{2}}-d_{2}\left(x_{p}+L / 2\right)}{y_{p}-d_{1}-d-2 d_{2}} \\
+ & \left(\left(x_{T_{2}}-x_{p}-\frac{L}{2}\right) \cdot\left(y_{p}-d_{1}-d-d_{2}\right)\right. \\
& \cdot\left[\left(d_{1}^{2}+2 d d_{1}+2 d_{2} d_{1}\right) \sqrt{\varepsilon}\right. \\
& \left.\left.+d^{2}+2 d_{2} d-\left(d_{1} \sqrt{\varepsilon}+d\right) y_{p}\right]\right) \\
& \times\left(\left[\sqrt{\varepsilon}\left(2 d_{1}+d+2 d_{2}-y_{p}\right)\right.\right. \\
& \left.\times\left(d_{1}+2 d+2 d_{2}-y_{p}\right)-d d_{1}\right] \\
& \left.\times\left(y_{p}-d_{1}-d-2 d_{2}\right)\right)^{-1}
\end{aligned}
$$

Using set $\Pi_{\mathrm{MIMO}}$ to denote the total obscured area, as in the above analysis, set $\Pi_{\mathrm{MIMO}}$ is

$$
\Pi_{\mathrm{MIMO}}=\Pi_{11}+\Pi_{12}+\Pi_{21}+\Pi_{22} \text {. }
$$

It is noted that set $\Pi_{\mathrm{MIMO}}$ is a function of transmitter positions and target position. Besides, signal distance in the first case is smaller than that in the second case. Furthermore, reflections from the obscured area in $\Pi_{12}$ and $\Pi_{22}$ will be scattered by the target and then received by other antennas. So in real application, EM is mainly obscured by the target in the first case, that is, $\Pi_{11}$ and $\Pi_{21}$.

Therefore, reflection from the back wall is

$$
\begin{array}{r}
s_{w}^{b}\left(t, \mathbf{x}_{R}\right)=\mathrm{FT}_{t \leftrightarrow k}^{-1}\left\{\sum _ { i = 1 } ^ { 2 } \left[A_{w}^{\prime} \cdot Z_{i}\left(k_{m}\right) \cdot \Gamma\left(\mathbf{x}_{T_{i}}, \mathbf{x}_{A_{3}^{\prime \prime}}, k_{m}\right)\right.\right. \\
\cdot \Lambda_{\text {exterior }}\left(\mathbf{x}_{T_{i}}, \mathbf{x}_{A_{3}^{\prime \prime}}, \mathbf{x}_{R}, k_{m}\right) \\
\cdot \Gamma\left(\mathbf{x}_{A_{3}^{\prime \prime}}, \mathbf{x}_{R}, k_{m}\right) \\
\left.\left.\cdot \exp \left(-j k_{m} \Delta l_{b}\left(\mathbf{x}_{T_{i}}, \mathbf{x}_{A_{3}^{\prime \prime}}, \mathbf{x}_{R}\right)\right)\right]\right\}, \\
\mathbf{x}_{A_{3}^{\prime \prime}} \notin \Pi_{\mathrm{MIMO}},
\end{array}
$$

where $s_{w}^{b}\left(t, \mathbf{x}_{R}\right)$ is reflections from the back wall received by antenna $R . \mathbf{x}_{A_{3}^{\prime \prime}}$ is the reflection position on the back wall. $\Delta l_{b}(\cdot)$ denotes signal distance of the back wall reflections. Taking $T_{1}$ in Figure 2(a), for example, the expression of $\Delta l_{b}(\cdot)$ is

$$
\begin{aligned}
\Delta l_{b}\left(\mathbf{x}_{T_{1}}, \mathbf{x}_{A_{3}^{\prime \prime}}, \mathbf{x}_{R_{7}}\right)= & \left\|\mathbf{x}_{T_{1}} \mathbf{x}_{A_{3}}\right\|+\left\|\mathbf{x}_{A_{3}} \mathbf{x}_{A_{3}^{\prime}}\right\| \cdot \sqrt{\varepsilon} \\
& +\left\|\mathbf{x}_{A_{3}^{\prime}} \mathbf{x}_{A_{3}^{\prime \prime}}\right\|+\left\|\mathbf{x}_{A_{3}^{\prime \prime}} \mathbf{x}_{A_{4}^{\prime}}\right\| \\
& +\left\|\mathbf{x}_{A_{4}^{\prime}} \mathbf{x}_{A_{4}}\right\| \cdot \sqrt{\varepsilon}+\left\|\mathbf{x}_{A_{4}} \mathbf{x}_{R_{7}}\right\|,
\end{aligned}
$$

where $\mathbf{x}_{A_{3}}$ and $\mathbf{x}_{A_{3}^{\prime}}$ are refraction positions on air-wall interface and wall-air interface of the front wall after signal transmitted by antenna $T_{1}$, respectively. $\mathbf{x}_{A_{4}^{\prime}}$ and $\mathbf{x}_{A_{4}}$ are refraction positions on air-wall interface and wall-air interface of the front wall before signal received by the receiver, respectively.

(B) Monostatic Through-the-Wall Mode. Figure 2(b) demonstrates the signal model for monostatic through-the-wall mode when an extended target is in a four-wall room. In this mode, reflection from the front wall is the same as (10). According to the extended target theory, the extended target scattering is

$$
\begin{gathered}
s_{g}\left(t, \mathbf{x}_{T R}\right)=\mathrm{FT}_{t \leftrightarrow k}^{-1}\left\{\sum_{n=1}^{N} A_{g}\left(\mathbf{x}_{U_{n}}\right) \cdot Z\left(k_{m}\right) \cdot \Gamma^{2}\left(\mathbf{x}_{T R}, \mathbf{x}_{U_{n}}, k_{m}\right)\right. \\
\left.\cdot \exp \left(-j k_{m} \Delta l_{g}\left(\mathbf{x}_{T R}, \mathbf{x}_{U_{n}}\right)\right)\right\} .
\end{gathered}
$$

As shown in the figure, the obscured area is different from that in MIMO mode. Obscured area $\Pi_{\text {monos. }}$ on the back wall conforms to

$$
\begin{aligned}
& \Pi_{\text {monos. }} \\
& = \begin{cases}x_{\mathrm{ob}} \in\left[\begin{array}{ll}
x_{P_{H}} & x_{P_{H}^{\prime}}
\end{array}\right] & x_{p}-\frac{L}{2}<x_{T R}<x_{p}+\frac{L}{2}, \\
y_{\mathrm{ob}}=d_{1}+d+d_{2}+y_{T_{1}}\end{cases}
\end{aligned}
$$

$$
\begin{aligned}
& x_{P_{H}}=x_{p}-\frac{L}{2}+(\left(x_{p}-\frac{L}{2}-x_{T R}\right) \\
& \times\left(\sqrt{\varepsilon} y_{p}-\sqrt{\varepsilon} y_{T R}-\sqrt{\varepsilon} d-d\right) \\
&\left.\times\left(y_{T R}+d_{1}+d+d_{2}-y_{p}\right)\right) \\
& \times\left(\sqrt{\varepsilon}\left(y_{p}-y_{T R}-d\right)\left(y_{p}-y_{T R}-d_{1}\right)-d d_{1}\right)^{-1}, \\
& x_{p}-\frac{L}{2}<x_{T R}<x_{p}+\frac{L}{2},
\end{aligned}
$$

$$
\begin{aligned}
x_{P_{H}^{\prime}}=x_{p}+\frac{L}{2}+( & \left(x_{p}+\frac{L}{2}-x_{T R}\right) \\
& \times\left(\sqrt{\varepsilon} y_{p}-\sqrt{\varepsilon} y_{T R}-\sqrt{\varepsilon} d-d\right) \\
& \left.\times\left(y_{T R}+d_{1}+d+d_{2}-y_{p}\right)\right) \\
\times\left(\sqrt{\varepsilon}\left(y_{p}-y_{T R}-d\right)\left(y_{p}-y_{T R}-d_{1}\right)-d d_{1}\right)^{-1}, & x_{p}-\frac{L}{2}<x_{T R}<x_{p}+\frac{L}{2} .
\end{aligned}
$$


Therefore, the back wall reflection is

$$
\begin{array}{r}
s_{w}^{b}\left(t, \mathbf{x}_{T R}\right)=\mathrm{FT}_{t \leftrightarrow k}^{-1}\left\{A_{w}^{\prime} \cdot Z\left(k_{m}\right) \cdot \Gamma^{2}\left(\mathbf{x}_{T}, \mathbf{x}_{A_{3}^{\prime \prime}}, k_{m}\right)\right. \\
\cdot \Lambda_{\text {exterior }}\left(\mathbf{x}_{T R}, \mathbf{x}_{A_{3}^{\prime \prime}}, k_{m}\right) \\
\left.\cdot \exp \left(-j k_{m} \Delta l_{b}\left(\mathbf{x}_{T}, \mathbf{x}_{A_{3}^{\prime \prime}}\right)\right)\right\}, \\
\mathbf{x}_{A_{3}^{\prime \prime}} \notin \Pi_{\text {monos. }},
\end{array}
$$

where the signal distance of back wall reflection is

$$
\Delta l_{b}\left(\mathbf{x}_{T}, \mathbf{x}_{A_{3}^{\prime \prime}}\right)=2\left(d_{1}+d \sqrt{\varepsilon}+d_{2}\right) .
$$

2.3. TWI Signal Model for Moving Extended Target. We assume that an extended target moves with constant speed $\vec{v}$. At time $t_{0}=0$, the target is located at $\mathbf{x}_{p}\left(t_{0}\right)$, with $\mathbf{x}_{p}\left(t_{0}\right)=\left(x_{p}\left(t_{0}\right), y_{p}\left(t_{0}\right)\right)$. At time $t$, the target moves to a new position, namely, $\mathbf{x}_{p}(t)=\left(x_{p}(t), y_{p}(t)\right)$. For convenience in the following derivation, we define $v_{x}$ and $v_{y}$ as the velocity components along the cross range axis and the range axis, respectively:

$$
\begin{aligned}
& x_{p}(t)=x_{p}\left(t_{0}\right)+v_{x} t, \\
& y_{p}(t)=y_{p}\left(t_{0}\right)+v_{y} t .
\end{aligned}
$$

(A) MIMO Through-the-Wall Mode. When the extended target moves in MIMO mode, target scattering is computed as

$$
\begin{aligned}
s_{g}\left(t, \mathbf{x}_{R}\right) & \\
=\mathrm{FT}_{t \leftrightarrow k}^{-1}\left\{\sum_{n=1}^{N} \sum_{i=1}^{2}[\right. & A_{g}\left(\mathbf{x}_{U_{n}(t)}\right) \cdot Z_{i}\left(k_{m}\right) \\
\cdot \Gamma\left(\mathbf{x}_{T_{i}}, \mathbf{x}_{U_{n}(t)}, k_{m}\right) & \\
\cdot \Gamma\left(\mathbf{x}_{U_{n}(t)}, \mathbf{x}_{R}, k_{m}\right) & \\
\cdot & \left.\left.\exp \left(-j k_{m} \Delta l_{g}\left(\mathbf{x}_{T_{i}}, \mathbf{x}_{U_{n}(t)}, \mathbf{x}_{R}\right)\right)\right]\right\},
\end{aligned}
$$

where variable $\mathbf{x}_{U_{n}(t)}$ is the position of the $n$th scattering point of the extended target at time $t$.

When target moves in this room, reflection from the first wall is the same as (6). However, the back wall reflection is different because the obscured area varies with target movement. In this model, the obscured area on the back wall at time $t$ is

$$
\Pi_{\mathrm{MIMO}}(t)=\Pi_{11}(t)+\Pi_{12}(t)+\Pi_{21}(t)+\Pi_{22}(t) .
$$

Therefore, reflection from the back wall can be derived:

$$
\begin{array}{r}
s_{w}^{b}\left(t, \mathbf{x}_{R}\right)=\mathrm{FT}_{t \leftrightarrow k}^{-1}\left\{\sum _ { i = 1 } ^ { 2 } \left[A_{w}^{\prime} \cdot Z_{i}\left(k_{m}\right) \cdot \Gamma\left(\mathbf{x}_{T_{i}}, \mathbf{x}_{A_{3}^{\prime \prime}}, k_{m}\right)\right.\right. \\
\cdot \Lambda_{\text {exterior }}\left(\mathbf{x}_{T_{i}}, \mathbf{x}_{A_{3}^{\prime \prime}}, \mathbf{x}_{R}, k_{m}\right) \\
\cdot \Gamma\left(\mathbf{x}_{A_{3}^{\prime \prime}}, \mathbf{x}_{R}, k_{m}\right) \\
\left.\left.\cdot \exp \left(-j k_{m} \Delta l_{b}\left(\mathbf{x}_{T_{i}}, \mathbf{x}_{A_{3}^{\prime \prime}}, \mathbf{x}_{R}\right)\right)\right]\right\},
\end{array}
$$$$
\mathbf{x}_{A_{3}^{\prime \prime}} \notin \Pi_{\mathrm{MIMO}}(t) \text {. }
$$

So, when an extended target moves in a four-wall room, the total echo received by antenna $R$ at time $t$ is

$$
\begin{aligned}
& S\left(t, \mathbf{x}_{R}\right) \\
& =\mathrm{FT}_{t \leftrightarrow k}^{-1}\left\{\sum _ { i = 1 } ^ { 2 } \left[A_{w} \cdot Z_{i}\left(k_{m}\right) \cdot \Lambda_{\text {exterior }}\left(\mathbf{x}_{T_{i}}, \mathbf{x}_{R}, k_{m}\right)\right.\right. \\
& \cdot \exp \left(-j k_{m} \Delta l_{1}^{f}\left(\mathbf{x}_{T_{i}}, \mathbf{x}_{R}\right)\right) \\
& +A_{w} \cdot Z_{i}\left(k_{m}\right) \cdot \Lambda_{\text {interior }}\left(\mathbf{x}_{T_{i}}, \mathbf{x}_{R}, k_{m}\right) \\
& \left.\left.\cdot \exp \left(-j k_{m} \Delta l_{2}^{f}\left(\mathbf{x}_{T_{i}}, \mathbf{x}_{R}\right)\right)\right]\right\} \\
& +\mathrm{FT}_{t \leftrightarrow k}^{-1}\left\{\sum _ { n = 1 } ^ { N } \sum _ { i = 1 } ^ { 2 } \left[A_{g}\left(\mathbf{x}_{U_{n}(t)}\right) \cdot Z_{i}\left(k_{m}\right)\right.\right. \\
& \cdot \Gamma\left(\mathbf{x}_{T_{i}}, \mathbf{x}_{U_{n}(t)}, k_{m}\right) \\
& \cdot \Gamma\left(\mathbf{x}_{U_{n}(t)}, \mathbf{x}_{R}, k_{m}\right) \\
& \left.\left.\cdot \exp \left(-j k_{m} \Delta l_{g}\left(\mathbf{x}_{T_{i}}, \mathbf{x}_{U_{n}(t)}, \mathbf{x}_{R}\right)\right)\right]\right\} \\
& +\mathrm{FT}_{t \leftrightarrow k}^{-1}\left\{\sum _ { i = 1 } ^ { 2 } \left[A_{w}^{\prime} \cdot Z_{i}\left(k_{m}\right) \cdot \Gamma\left(\mathbf{x}_{T_{i}}, \mathbf{x}_{A_{3}^{\prime \prime}}, k_{m}\right)\right.\right. \\
& \cdot \Lambda_{\text {exterior }}\left(\mathbf{x}_{T_{i}}, \mathbf{x}_{A_{3}^{\prime \prime}}, \mathbf{x}_{R}, k_{m}\right) \\
& \cdot \Gamma\left(\mathbf{x}_{A_{3}^{\prime \prime}}, \mathbf{x}_{R}, k_{m}\right) \\
& \left.\left.\cdot \exp \left(-j k_{m} \Delta l_{b}\left(\mathbf{x}_{T_{i}}, \mathbf{x}_{A_{3}^{\prime \prime}}, \mathbf{x}_{R}\right)\right)\right]\right\} \text {, } \\
& A_{3}^{\prime \prime} \notin \Pi_{\mathrm{MIMO}}(t) \text {. }
\end{aligned}
$$


(B) Monostatic Through-the-Wall Mode. When the extended target moves in monostatic mode, target scattering is computed as

$$
\begin{aligned}
s_{g}\left(t, \mathbf{x}_{T R}\right)=\mathrm{FT}_{t \leftrightarrow k}^{-1}\left\{\sum_{n=1}^{N} A_{g}\left(\mathbf{x}_{U_{n}(t)}\right) \cdot Z\left(k_{m}\right)\right. \\
\cdot \Gamma^{2}\left(\mathbf{x}_{T R}, \mathbf{x}_{U_{n}(t)}, k_{m}\right) \\
\left.\cdot \exp \left(-j k_{m} \Delta l_{g}\left(\mathbf{x}_{T R}, \mathbf{x}_{U_{n}(t)}\right)\right)\right\} .
\end{aligned}
$$

Target movement does not affect the reflection from the first wall, which is denoted by (10). However, the obscured area varies with time $t$. So reflections from the back wall are

$$
\begin{array}{r}
s_{w}^{b}\left(t, \mathbf{x}_{T R}\right)=\mathrm{FT}_{t \leftrightarrow k}^{-1}\left\{A_{w}^{\prime} \cdot Z\left(k_{m}\right) \cdot \Gamma^{2}\left(\mathbf{x}_{T R}, \mathbf{x}_{A_{3}^{\prime \prime}}, k_{m}\right)\right. \\
\cdot \Lambda_{\text {exterior }}\left(\mathbf{x}_{T R}, \mathbf{x}_{A_{3}^{\prime \prime}}, k_{m}\right) \\
\left.\cdot \exp \left(-j k_{m} \Delta l_{b}\left(\mathbf{x}_{T R}, \mathbf{x}_{A_{3}^{\prime \prime}}\right)\right)\right\}, \\
\mathbf{x}_{A_{3}^{\prime \prime}} \notin \Pi_{\text {monos. }}(t) .
\end{array}
$$

As a result, in monostatic through-the-wall mode, the total echo received by antenna $R$ at time $t$ is

$$
\begin{aligned}
& S\left(t, \mathbf{x}_{T R}\right)=\mathrm{FT}_{t \leftrightarrow k}^{-1}\left\{A_{w} \cdot Z\left(k_{m}\right) \cdot \Lambda_{\text {exterior }}\left(\mathbf{x}_{T R}, k_{m}\right)\right. \\
& \cdot \exp \left(-j k_{m} \Delta l_{1}^{f}\left(\mathbf{x}_{T R}\right)\right) \\
& +A_{w} \cdot Z\left(k_{m}\right) \cdot \Lambda_{\text {interior }}\left(\mathbf{x}_{T R}, k_{m}\right) \\
& \left.\cdot \exp \left(-j k_{m} \Delta l_{2}^{f}\left(\mathbf{x}_{T R}\right)\right)\right\} \\
& +\mathrm{FT}_{t \leftrightarrow k}^{-1}\left\{\sum_{n=1}^{N} A_{g}\left(\mathbf{x}_{U_{n}(t)}\right) \cdot Z\left(k_{m}\right)\right. \\
& \cdot \Gamma^{2}\left(\mathbf{x}_{T R}, \mathbf{x}_{U_{n}(t)}, k_{m}\right) \\
& \left.\cdot \exp \left(-j k_{m} \Delta l_{g}\left(\mathbf{x}_{T R}, \mathbf{x}_{U_{n}(t)}\right)\right)\right\} \\
& +\mathrm{FT}_{t \leftrightarrow k}^{-1}\left\{A_{w}^{\prime} \cdot Z\left(k_{m}\right) \cdot \Gamma^{2}\left(\mathbf{x}_{T R}, \mathbf{x}_{A_{3}^{\prime \prime}}, k_{m}\right)\right. \\
& \cdot \Lambda_{\text {exterior }}\left(\mathbf{x}_{T R}, \mathbf{x}_{A_{3}^{\prime \prime}}, k_{m}\right) \\
& \left.\cdot \exp \left(-j k_{m} \Delta l_{b}\left(\mathbf{x}_{T R}, \mathbf{x}_{A_{3}^{\prime \prime}}\right)\right)\right\}, \\
& A_{3}^{\prime \prime} \notin \Pi_{\text {monos. }}(t) \text {. }
\end{aligned}
$$

\section{Moving Target Imaging and Ghost Analysis}

Compared with the conventional point target signal models, new characteristics can be concluded when the target is an extended one. (i) The extended target, such as human, obscures the EM propagation. As a result, reflection from the back wall changes. (ii) The obscured area varies with target movement. Therefore, the moving target imaging results will be affected. In this section, we will analyze the effect of target obscuring on moving target imaging and propose a method to remove or minimize these effects.

In moving target imaging, change detection method is the first step because of its efficiency in removing the strong clutter and noise. The change detection operation is

$$
\Delta S\left(t, \mathbf{x}_{R}\right)=S\left(t+\Delta t, \mathbf{x}_{R}\right)-S\left(t, \mathbf{x}_{R}\right),
$$

where $\Delta S(\cdot)$ is the subtraction result after change detection.

Then we make imaging processing for the subtract result, $\Delta S\left(t, \mathbf{x}_{R}\right)$. Here we adopt the back projection (BP) imaging method [18-20]. In the imaging operation, the region of interest is divided into a finite number of pixels in range and cross range directions. The complex amplitude image value for the pixel located at $\mathbf{x}_{q}=\left(x_{q}, y_{q}\right)$ is obtained by applying frequency-dependent phase and weights to all the received data:

$$
\begin{aligned}
& I\left(x_{q}, y_{q}\right) \\
& =\iint w \cdot \Delta S\left(t, \mathbf{x}_{R}\right) \cdot \exp \left(\frac{j 2 \pi f_{m} r\left(\mathbf{x}_{T}, \mathbf{x}_{q}, \mathbf{x}_{R}\right)}{c}\right) d \mathbf{x}_{T} d \mathbf{x}_{R},
\end{aligned}
$$

where $w$ is the weighting function to shape the beam. $r\left(\mathbf{x}_{T}, \mathbf{x}_{q}, \mathbf{x}_{R}\right)$ is the compensation signal distance for the pixel $\mathbf{x}_{q}$. It is computed as

$$
\begin{aligned}
r\left(\mathbf{x}_{T}, \mathbf{x}_{q}, \mathbf{x}_{R}\right)= & \sqrt{\left(x_{T}-x_{q}\right)^{2}+\left(y_{T}-y_{q}\right)^{2}} \\
& +\sqrt{\left(x_{q}-x_{R}\right)^{2}+\left(y_{q}-y_{R}\right)^{2}} .
\end{aligned}
$$

According to change detection and BP method, moving target imaging for extended target in MIMO and monostatic modes can be computed.

(A) MIMO Through-the-Wall Mode. By using (30), the subtract result, $\Delta S\left(t, \mathbf{x}_{R}\right)$, after change detection is

$$
\begin{aligned}
& \Delta S\left(t, \mathbf{x}_{R}\right) \\
& =S\left(t+\Delta t, \mathbf{x}_{R}\right)-S\left(t, \mathbf{x}_{R}\right) \\
& =\left\{\mathrm{FT}_{t \leftrightarrow k}^{-1}\left\{\sum_{n=1}^{N} \sum_{i=1}^{2}\left[A_{g}\left(\mathbf{x}_{U_{n}(t+\Delta t)}\right) \cdot Z_{i}\left(k_{m}\right) \cdot \Gamma\left(\mathbf{x}_{T_{i}}, \mathbf{x}_{U_{n}(t+\Delta t)}, k_{m}\right) \cdot \Gamma\left(\mathbf{x}_{U_{n}(t+\Delta t)}, \mathbf{x}_{R}, k_{m}\right) \cdot \exp \left(-j k_{m} \Delta l_{g}\left(\mathbf{x}_{T_{i}}, \mathbf{x}_{U_{n}(t+\Delta t)}, \mathbf{x}_{R}\right)\right)\right]\right\}\right.
\end{aligned}
$$




$$
\begin{aligned}
& \left.-\mathrm{FT}_{t \leftrightarrow k}^{-1}\left\{\sum_{n=1}^{N} \sum_{i=1}^{2}\left[A_{g}\left(\mathbf{x}_{U_{n}(t)}\right) \cdot Z_{i}\left(k_{m}\right) \cdot \Gamma\left(\mathbf{x}_{T_{i}}, \mathbf{x}_{U_{n}(t)}, k_{m}\right) \cdot \Gamma\left(\mathbf{x}_{U_{n}(t)}, \mathbf{x}_{R}, k_{m}\right) \cdot \exp \left(-j k_{m} \Delta l_{g}\left(\mathbf{x}_{T_{i}}, \mathbf{x}_{U_{n}(t)}, \mathbf{x}_{R}\right)\right)\right]\right\}\right\} \\
+ & \underbrace{\left\{\mathrm{FT}_{t \leftrightarrow k}^{-1}\left\{A_{w}^{\prime} \cdot Z_{1}\left(k_{m}\right) \cdot \Gamma\left(\mathbf{x}_{T_{1}}, \mathbf{x}_{A_{3}^{\prime \prime}}, k_{m}\right) \cdot \Lambda_{\text {exterior }}\left(\mathbf{x}_{T_{1}}, \mathbf{x}_{A_{3}^{\prime \prime}}, \mathbf{x}_{R}, k_{m}\right) \cdot \Gamma\left(\mathbf{x}_{A_{3}^{\prime \prime}}, \mathbf{x}_{R}, k_{m}\right) \cdot \exp \left(-j k_{m} \Delta l_{b}\left(\mathbf{x}_{T_{1}}, \mathbf{x}_{A_{3}^{\prime \prime}}, \mathbf{x}_{R}\right)\right)\right\}\right\}}_{A_{3}^{\prime \prime} \in\left(\bar{\Pi}_{1}(t+\Delta t)-\bar{\Pi}_{1}(t)\right) \cup\left(\bar{\Pi}_{1}(t)-\bar{\Pi}_{1}(t+\Delta t)\right)} \\
+ & \underbrace{\left\{\mathrm{FT}_{t \leftrightarrow k}^{-1}\left\{A_{w}^{\prime} \cdot Z_{2}\left(k_{m}\right) \cdot \Gamma\left(\mathbf{x}_{T_{2}}, \mathbf{x}_{A_{3}^{\prime \prime}}, k_{m}\right) \cdot \Lambda_{\text {exterior }}\left(\mathbf{x}_{T_{2}}, \mathbf{x}_{A_{3}^{\prime \prime}}, \mathbf{x}_{R}, k_{m}\right) \cdot \Gamma\left(\mathbf{x}_{A_{3}^{\prime \prime}}, \mathbf{x}_{R}, k_{m}\right) \cdot \exp \left(-j k_{m} \Delta l_{b}\left(\mathbf{x}_{T_{2}}, \mathbf{x}_{A_{3}^{\prime \prime}}, \mathbf{x}_{R}\right)\right)\right\}\right\}}_{A_{3}^{\prime \prime} \in\left(\bar{\Pi}_{2}(t+\Delta t)-\bar{\Pi}_{2}(t)\right) \cup\left(\bar{\Pi}_{2}(t)-\bar{\Pi}_{2}(t+\Delta t)\right)},
\end{aligned}
$$

where the first term in (37) is the subtraction result for target scattering. The second and the third terms denote the subtraction result for the back wall reflection when $T_{1}$ and $T_{2}$ work, respectively. Two sets, $\Pi_{1}(\cdot)$ and $\Pi_{2}(\cdot)$, denote the obscured areas corresponding to transmitter $T_{1}$ and transmitter $T_{2}$, respectively. Referring to (15a), (15b), (15c), (16a), (16b), (16c), (17a), (17b), (17c), (18a), (18b), and (18c), expressions of the two sets are

$$
\begin{aligned}
& \Pi_{1}(t)=\Pi_{11}(t)+\Pi_{12}(t), \\
& \Pi_{2}(t)=\Pi_{21}(t)+\Pi_{22}(t) .
\end{aligned}
$$

After BP imaging, there are two types of targets in the result; namely,

$$
\begin{aligned}
& I\left(x_{q}, y_{q}\right) \\
& =I_{\text {target }}\left(x_{q}, y_{q}\right)+G_{T_{1}}\left(x_{q}, y_{q}\right)+G_{T_{2}}\left(x_{q}, y_{q}\right) \\
& =\iint\left\{\mathrm{FT}_{t \leftrightarrow k}^{-1}\left\{\sum_{n=1}^{N} \sum_{i=1}^{2}\left[A_{g}\left(\mathbf{x}_{U_{n}(t+\Delta t)}\right) \cdot Z_{i}\left(k_{m}\right) \cdot \Gamma\left(\mathbf{x}_{T_{i}}, \mathbf{x}_{U_{n}(t+\Delta t)}, k_{m}\right) \cdot \Gamma\left(\mathbf{x}_{U_{n}(t+\Delta t)}, \mathbf{x}_{R}, k_{m}\right) \cdot \exp \left(-j k_{m} \Delta l_{g}\left(\mathbf{x}_{T_{i}}, \mathbf{x}_{U_{n}(t+\Delta t)}, \mathbf{x}_{R}\right)\right)\right]\right\}\right. \\
& \left.-\mathrm{FT}_{t \leftrightarrow k}^{-1}\left\{\sum_{n=1}^{N} \sum_{i=1}^{2}\left[A_{g}\left(\mathbf{x}_{U_{n}(t)}\right) \cdot Z_{i}\left(k_{m}\right) \cdot \Gamma\left(\mathbf{x}_{T_{i}}, \mathbf{x}_{U_{n}(t)}, k_{m}\right) \cdot \Gamma\left(\mathbf{x}_{U_{n}(t)}, \mathbf{x}_{R}, k_{m}\right) \cdot \exp \left(-j k_{m} \Delta l_{g}\left(\mathbf{x}_{T_{i}}, \mathbf{x}_{U_{n}(t)}, \mathbf{x}_{R}\right)\right)\right]\right\}\right\} \\
& \times \exp \left(\frac{j 2 \pi f_{m} r\left(\mathbf{x}_{T_{i}}, \mathbf{x}_{q}, \mathbf{x}_{R}\right)}{c}\right) d \mathbf{x}_{T_{i}} d \mathbf{x}_{R} \\
& +\iint \underbrace{\left\{\mathrm{FT}_{t \leftrightarrow k}^{-1}\left\{A_{w}^{\prime} \cdot Z_{1}\left(k_{m}\right) \cdot \Gamma\left(\mathbf{x}_{T_{1}}, \mathbf{x}_{A_{3}^{\prime \prime}}, k_{m}\right) \cdot \Lambda_{\text {exterior }}\left(\mathbf{x}_{T_{1}}, \mathbf{x}_{A^{\prime \prime}}, \mathbf{x}_{R}, k_{m}\right) \cdot \Gamma\left(\mathbf{x}_{A^{\prime \prime}}, \mathbf{x}_{R}, k_{m}\right) \cdot \exp \left(-j k_{m} \Delta l_{b}\left(\mathbf{x}_{T_{1}}, \mathbf{x}_{A_{3}^{\prime \prime}}, \mathbf{x}_{R}\right)\right)\right\}\right\}}_{A_{3}^{\prime \prime} \in\left(\bar{\Pi}_{1}(t+\Delta t)-\bar{\Pi}_{1}(t)\right) \cup\left(\bar{\Pi}_{1}(t)-\bar{\Pi}_{1}(t+\Delta t)\right)} \\
& \times \underbrace{\exp \left(\frac{j 2 \pi f_{m} r\left(\mathbf{x}_{T_{1}}, \mathbf{x}_{q}, \mathbf{x}_{R}\right)}{c}\right)}_{A_{3}^{\prime \prime} \in\left(\bar{\Pi}_{1}(t+\Delta t)-\bar{\Pi}_{1}(t)\right) \cup\left(\bar{\Pi}_{1}(t)-\bar{\Pi}_{1}(t+\Delta t)\right)} d \mathbf{x}_{R} \\
& +\iint \underbrace{\left\{\mathrm{FT}_{t \leftrightarrow k}^{-1}\left\{A_{w}^{\prime} \cdot Z_{2}\left(k_{m}\right) \cdot \Gamma\left(\mathbf{x}_{T_{2}}, \mathbf{x}_{A_{3}^{\prime \prime}}, k_{m}\right) \cdot \Lambda_{\text {exterior }}\left(\mathbf{x}_{T_{2}}, \mathbf{x}_{A^{\prime \prime}}, \mathbf{x}_{R}, k_{m}\right) \cdot \Gamma\left(\mathbf{x}_{A^{\prime \prime}}, \mathbf{x}_{R}, k_{m}\right) \cdot \exp \left(-j k_{m} \Delta l_{b}\left(\mathbf{x}_{T_{2}}, \mathbf{x}_{A_{3}^{\prime \prime}}, \mathbf{x}_{R}\right)\right)\right\}\right\}}_{A_{3}^{\prime \prime} \in\left(\bar{\Pi}_{2}(t+\Delta t)-\bar{\Pi}_{2}(t)\right) \cup\left(\bar{\Pi}_{2}(t)-\bar{\Pi}_{2}(t+\Delta t)\right)} \\
& \times \underbrace{\exp \left(\frac{j 2 \pi f_{m} r\left(\mathbf{x}_{T_{2}}, \mathbf{x}_{q}, \mathbf{x}_{R}\right)}{c}\right)}_{A_{3}^{\prime \prime} \in\left(\overline{\mathbf{\Pi}}_{2}(t+\Delta t)-\overline{\mathbf{\Pi}}_{2}(t)\right) \cup\left(\overline{\mathbf{\Pi}}_{2}(t)-\bar{\Pi}_{2}(t+\Delta t)\right)} d \mathbf{x}_{R}
\end{aligned}
$$

where $I_{\text {target }}(\cdot)$ denotes the true target image. $G_{T_{1}}(\cdot)$ and $G_{T_{2}}(\cdot)$ are the ghosts corresponding to transmitter $T_{1}$ and transmitter $T_{2}$. It is noted that target obscuring is more severe in the first case than in the second case, so $G_{T_{1}}(\cdot)$ and $G_{T_{2}}(\cdot)$ 
are mainly contributed by target obscuring in the first case in real application.
(B) Monostatic Through-the-Wall Mode. By using change detection method, the subtract result in monostatic throughthe-wall mode is

$$
\begin{aligned}
& \Delta S\left(t, \mathbf{x}_{T R}\right) \\
& =S\left(t+\Delta t, \mathbf{x}_{T R}\right)-S\left(t, \mathbf{x}_{T R}\right) \\
& =\left\{\mathrm{FT}_{t \leftrightarrow k}^{-1}\left\{\sum_{n=1}^{N} A_{g}\left(\mathbf{x}_{U_{n}(t+\Delta t)}\right) \cdot Z\left(k_{m}\right) \cdot \Gamma^{2}\left(\mathbf{x}_{T R}, \mathbf{x}_{U_{n}(t+\Delta t)}, k_{m}\right) \cdot \exp \left(-j k_{m} \Delta l_{g}\left(\mathbf{x}_{T R}, \mathbf{x}_{U_{n}(t+\Delta t)}\right)\right)\right\}\right. \\
& \left.\quad-\mathrm{FT}_{t \leftrightarrow k}^{-1}\left\{\sum_{n=1}^{N} A_{g}\left(\mathbf{x}_{U_{n}(t)}\right) \cdot Z\left(k_{m}\right) \cdot \Gamma^{2}\left(\mathbf{x}_{T R}, \mathbf{x}_{U_{n}(t)}, k_{m}\right) \cdot \exp \left(-j k_{m} \Delta l_{g}\left(\mathbf{x}_{T R}, \mathbf{x}_{U_{n}(t)}\right)\right)\right\}\right\} \\
& +\underbrace{\mathrm{FT}_{t \leftrightarrow k}^{-1}\left\{A_{w}^{\prime} \cdot Z\left(k_{m}\right) \cdot \Gamma^{2}\left(\mathbf{x}_{T R}, \mathbf{x}_{A_{3}^{\prime \prime}}, k_{m}\right) \cdot \Lambda_{\text {exterior }}\left(\mathbf{x}_{T R}, \mathbf{x}_{A_{3}^{\prime \prime}}, k_{m}\right) \cdot \exp \left(-j k_{m} \Delta l_{b}\left(\mathbf{x}_{T R}, \mathbf{x}_{A_{3}^{\prime \prime}}\right)\right)\right\}}_{A_{3}^{\prime \prime} \in\left(\overline{\boldsymbol{\Pi}}_{\text {monos. }}(t+\Delta t)-\overline{\boldsymbol{\Pi}}_{\text {monos. }}(t)\right) \cup\left(\overline{\boldsymbol{\Pi}}_{\text {monos. }}(t)-\overline{\boldsymbol{\Pi}}_{\text {monos. }}(t+\Delta t)\right)} .
\end{aligned}
$$

Then the imaging result for an extended moving target is

$$
\begin{aligned}
& I\left(x_{q}, y_{q}\right) \\
& =I_{\text {target }}\left(x_{q}, y_{q}\right)+G\left(x_{q}, y_{q}\right) \\
& =\iint\left\{\mathrm{FT}_{t \leftrightarrow k}^{-1}\left\{\sum_{n=1}^{N} A_{g}\left(\mathbf{x}_{U_{n}(t+\Delta t)}\right) \cdot Z\left(k_{m}\right) \cdot \Gamma^{2}\left(\mathbf{x}_{T R}, \mathbf{x}_{U_{n}(t+\Delta t)}, k_{m}\right) \cdot \exp \left(-j k_{m} \Delta l_{g}\left(\mathbf{x}_{T R}, \mathbf{x}_{U_{n}(t+\Delta t)}\right)\right)\right\}\right. \\
& \left.\quad-\mathrm{FT}_{t \leftrightarrow k}^{-1}\left\{\sum_{n=1}^{N} A_{g}\left(\mathbf{x}_{U_{n}(t)}\right) \cdot Z\left(k_{m}\right) \cdot \Gamma^{2}\left(\mathbf{x}_{T R}, \mathbf{x}_{U_{n}(t)}, k_{m}\right) \cdot \exp \left(-j k_{m} \Delta l_{g}\left(\mathbf{x}_{T R}, \mathbf{x}_{U_{n}(t)}\right)\right)\right\}\right\} \exp \left(\frac{j 2 \pi f_{m} r\left(\mathbf{x}_{T R}, \mathbf{x}_{q}\right)}{c}\right) d \mathbf{x}_{T R} \\
& \quad+\int \underbrace{\operatorname{FT}_{t \leftrightarrow k}^{-1}\left\{A_{w}^{\prime} \cdot Z\left(k_{m}\right) \cdot \Gamma^{2}\left(\mathbf{x}_{T R}, \mathbf{x}_{A_{3}^{\prime \prime}}, k_{m}\right) \cdot \Lambda_{\text {exterior }}\left(\mathbf{x}_{T R}, \mathbf{x}_{A_{3}^{\prime \prime}}, k_{m}\right) \cdot \exp \left(-j k_{m} \Delta l_{b}\left(\mathbf{x}_{T R}, \mathbf{x}_{A_{3}^{\prime \prime}}\right)\right)\right\}}_{A_{3}^{\prime \prime} \in\left(\bar{\Pi}_{\text {monos. }}(t+\Delta t)-\bar{\Pi}_{\text {monos. }}(t)\right) \cup\left(\bar{\Pi}_{\text {monos. }}(t)-\overline{\mathbf{n}}_{\text {monos. }}(t+\Delta t)\right)} d \mathbf{x}_{T R},
\end{aligned}
$$

where $G(\cdot)$ is the ghost in monostatic through-the-wall mode.

It is mentioned that when EM wave propagates through the wall, the velocity will be slow; that is, $v=c / \sqrt{\varepsilon}$, which is determined by the electromagnetic parameters of the wall. In the processing by (41), the velocity is assumed to be propagating in free space; that is, $v=c$. Therefore, in the original imaging results, the target will be imaged behind its true position and the ghost will also be imaged behind the obscured area. The shift distance equals $(\sqrt{\varepsilon}-1) \cdot d$ in range direction. In this paper, all the following processing is applied based on the original imaging results.

\section{Ghost Mitigation in Through-the-Wall Moving Target Imaging}

According to the proposed signal models, some conclusions can be obtained. For one thing, ghosts have a spatial relationship with the targets and transmitters in the original imaging results. In MIMO mode, as shown in Figure 3, when ghost occurs in the first case, transmitters, target, and ghost are approximately on a line in the imaging result. When it occurs in the second case, mirror transmitter, ghost, and target are approximately on a line. In monostatic mode, as shown in Figure 4, the line of the target and ghost is approximately perpendicular to the antennas array. For another, ghosts are in essence the imaging residue of the back wall. So the values in range direction of their positions are constants.

Here an approach is proposed to remove the ghosts. Firstly, adopt CFAR algorithm to obtain the detected imaging result. Then extract spatial positions of all the targets via clustering method. Finally judge the ghosts and remove them according to the spatial geometric relationship.

(1) CFAR Detection. To detect targets and suppress the clutter, a Gauss distribution based CFAR method is adopted firstly. When CFAR detection is finished, a new image, $B$, is obtained, with $B=\left\{b_{i, j} \mid i=1, \ldots, M_{B}, j=1, \ldots, N_{B}\right\}$. Here, 


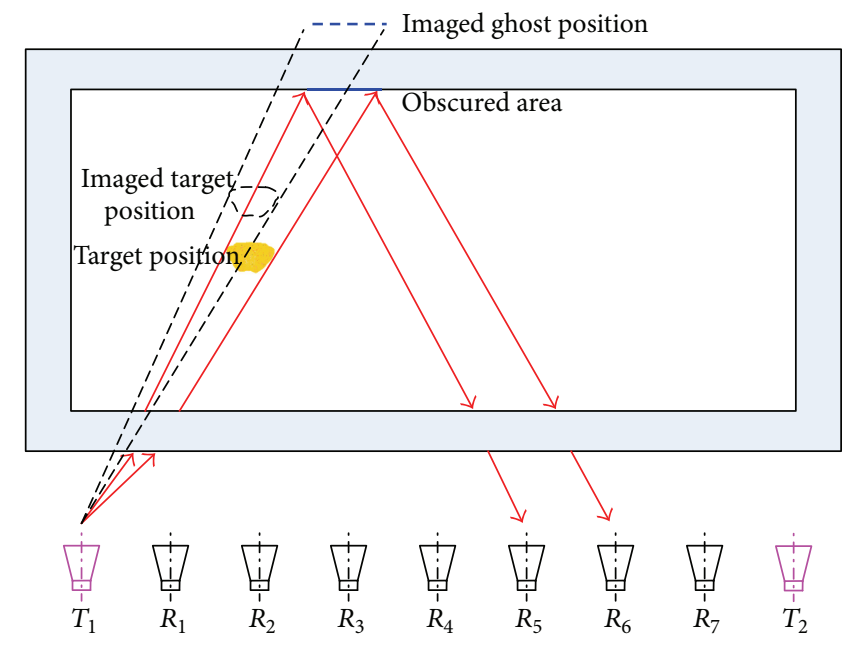

(a)

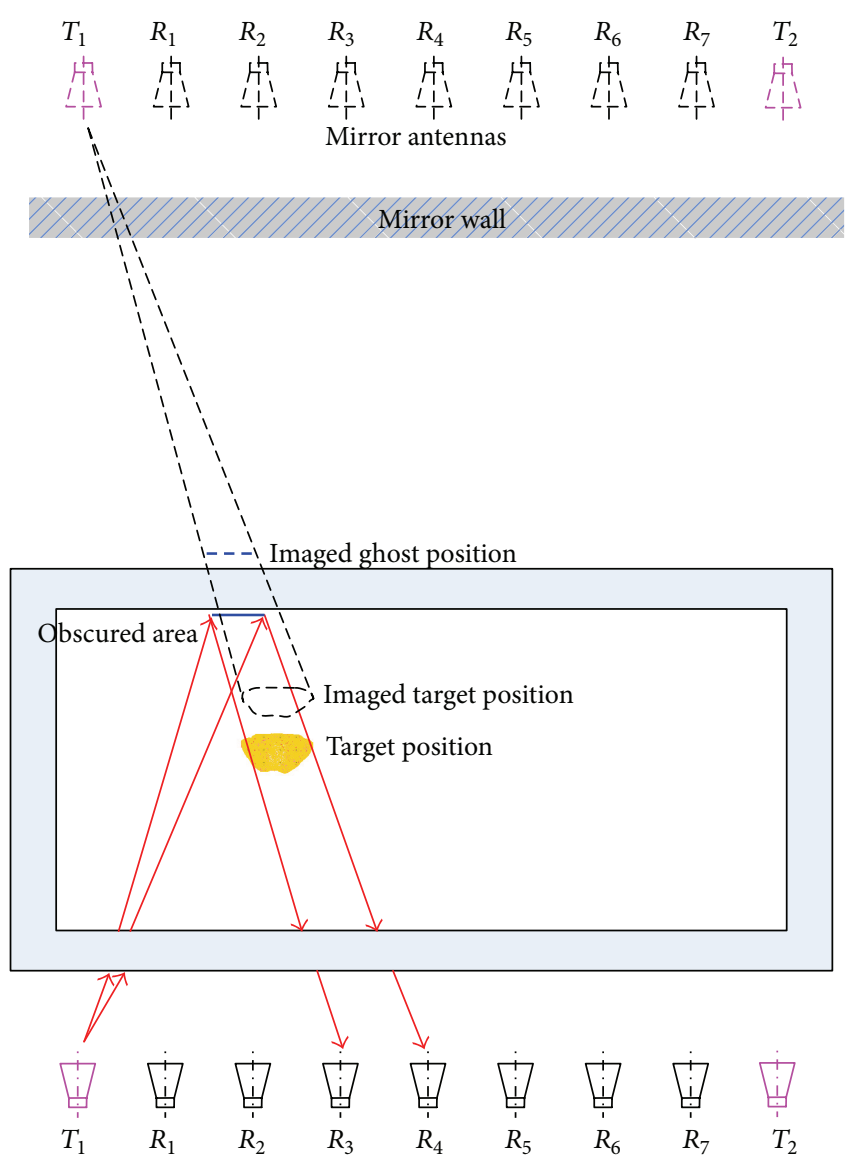

(b)

FIGURE 3: Spatial relationship among the antenna (or mirror antennas), target, and ghost in MIMO mode. (a) Ghost occurs in the first case. (b) Ghost occurs in the second case.

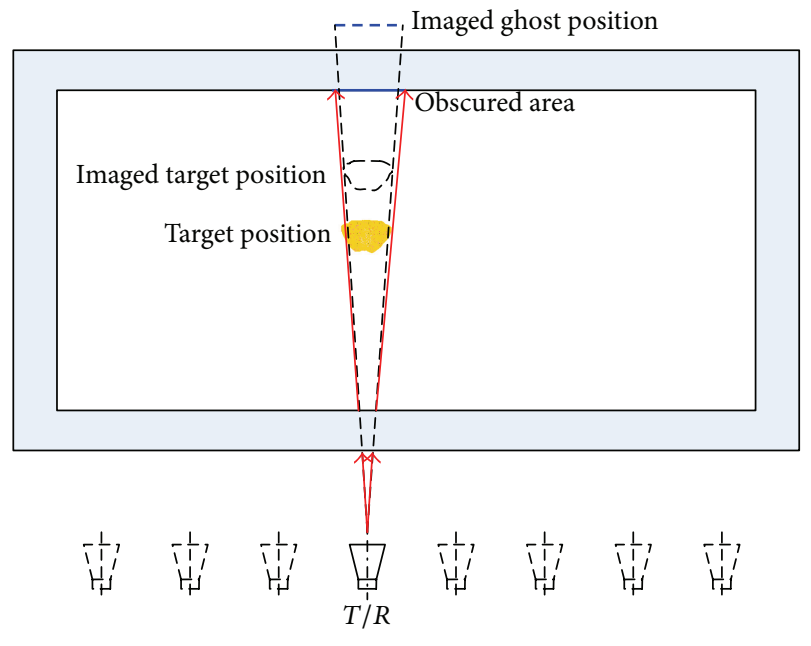

FIGURE 4: Geometric relationship among the antenna, target, and ghost in monostatic mode.

$M_{B}$ and $N_{B}$ are the number of pixels in range axis and cross range axis, respectively.
(2) Extract Spatial Positions via Clustering Method. By using the clustering method, spatial positions of all the targets can be extracted. During clustering processing, clustering centers are updated by iterative operation. They are computed by

$$
\begin{aligned}
& x_{l}^{\prime}=\frac{1}{k_{l}^{\prime}}\left\{x_{l} \times k_{l}+i \times\left[1+\frac{b_{q}}{\max \left(b_{q}\right)}\right]\right\}, \\
& y_{l}^{\prime}=\frac{1}{k_{l}^{\prime}}\left\{y_{l} \times k_{l}+j \times\left[1+\frac{b_{q}}{\max \left(b_{q}\right)}\right]\right\}, \\
& k_{l}^{\prime}=k_{l}+1+\frac{b_{i, j}}{\max \left(b_{i, j}\right)},
\end{aligned}
$$

where $\left(x_{l}, y_{l}\right)$ and $\left(x_{l}^{\prime}, y_{l}^{\prime}\right)$ are the position of the lth clustering center before and after the updating operation, respectively. $b_{q}$ denotes grey value of the pixel located at $\left(x_{q}, y_{q}\right) \cdot \max \left(b_{i j}\right)$ is the maximum grey value of all the pixels in the image. $k_{l}$ and 
$k_{l}^{\prime}$ denote the number of the $l$ th cluster before and after the updating operation, respectively.

(3) Ghosts Judgment and Mitigation. Steps for removing the ghost are as follows (taking the ith imaging result, for example).

Step 1. Detect the back wall in the $i$ th imaging result. Then calculate the symmetric positions of transmitters from the back wall. Transmitters and corresponding mirror transmitters are denoted by $\left(x_{T}^{r}, y_{T}^{r}\right)$ and $\left(x_{T^{\prime}}^{r}, y_{T^{\prime}}^{r}\right)$, respectively, where $r$ is the index, with $r=1,2, \ldots, N T$. Here NT is the number of the transmitters.

Using the change detection, CFAR, and clustering methods, extract spatial positions of all the targets and define them as $\mathbf{P}=\left(x_{p}^{i}, y_{p}^{i}\right)$, with $p=1,2, \ldots, N P$, where $N P$ denotes the number of the targets in the image.

Step 2. Read one of the targets in the image and define it as $P_{1}=\left(x_{p_{1}}^{i}, y_{p_{1}}^{i}\right)$, with $P_{1} \in \mathbf{P}$. Then read another target behind $P_{1}$, denoted by $P_{2}=\left(x_{p_{2}}^{i}, y_{p_{2}}^{i}\right)$, with $P_{2} \in \mathbf{P}$.

(a) If it is in the MIMO through-the-wall mode, then judge whether one of transmitters (or mirror transmitters) exists conforming to

$$
\begin{aligned}
& \left|\frac{y_{p_{2}}^{i}-y_{p_{1}}^{i}}{x_{p_{2}}^{i}-x_{p_{1}}^{i}}-\frac{y_{p_{1}}^{i}-y_{T}^{r}}{x_{p_{1}}^{i}-y_{T}^{r}}\right|<\delta \\
& \text { or }\left|\frac{y_{p_{2}}^{i}-y_{p_{1}}^{i}}{x_{p_{2}}^{i}-x_{p_{1}}^{i}}-\frac{y_{p_{1}}^{i}-y_{T^{\prime}}^{r}}{x_{p_{1}}^{i}-y_{T^{\prime}}^{r}}\right|<\delta, \\
& \quad r=1,2 \ldots, N T,
\end{aligned}
$$

where the variable $\delta$ is a threshold for judging whether the target, ghost, and transmitters (or mirror transmitter) are approximately on a line. If the transmitter or mirror transmitter can be found, then go to Step 3, or update variable $P_{2}$.

(b) If it is in the monostatic through-the-wall mode, judge whether the line of $P_{1}$ and $P_{2}$ is perpendicular to the antennas array

$$
\left|\frac{y_{p_{2}}^{i}-y_{p_{1}}^{i}}{x_{p_{2}}^{i}-x_{p_{1}}^{i}}-\frac{x_{T}^{r}-x_{T}^{r-1}}{y_{T}^{r}-y_{T}^{r-1}}\right|<\mu, \quad r=2,3, \ldots, N T,
$$

where the variable $\mu$ is the judgment threshold. If the line of $P_{1}$ and $P_{2}$ is perpendicular to the antennas array, then go to Step 3, or update variable $P_{2}$.

Step 3. Judge whether the position value in range direction of $P_{2}$ is unchanged according to

$$
\left|y_{p_{2}}^{i}-y_{p_{2}}^{i-1}\right| \leq \alpha, \quad i=2,3, \ldots, K,
$$

where the variable $\alpha$ is a threshold. $y_{p_{2}}^{i-1}$ denotes the position value in range direction of $P_{2}$ in the $(i-1)$ th imaging result. $K$ is the number of imaging results.
Step 4. If $P_{2}$ conforms to (43)-(45), then it can be determined that it is a ghost. Once it is determined, all the pixels in the cluster of $P_{2}$ are reset to zero, otherwise they are reset to one.

Step 5. Update $P_{1}$ and $P_{2}$ until all the targets have been checked according to Steps 2 to 4 .

At last, a new binary image is obtained, which is denoted by $I^{\prime}(x, y)$. In this image, pixels in the target cluster are set to one and pixels in ghost cluster are set to zero. Since $I^{\prime}(x, y)$ is a binary image, the final imaging result after ghost removed is computed as

$$
\widetilde{I}(x, y)=I(x, y) \otimes I^{\prime}(x, y),
$$

where $\widetilde{I}(x, y)$ denoted the final image after ghost removed. $I(x, y)$ is the original imaging result of moving target. The operation $\otimes$ denotes multiplication for each pixel.

\section{Electromagnetic Simulations and Experiments}

In this section, electromagnetic simulations and experiments are applied to validate the constructed signal model and the proposed method.

(A) Electromagnetic Simulation about Obscuring in EM Propagation. In order to analyze the effect of target on EM propagation, we employ the FDTD algorithm. FDTD is a full-wave computational EM algorithm based on discretizing Maxwell's time-domain equations, using finite differences in spatial and temporal dimensions. Because this algorithm operates in the time domain, results can be provided over a wide range of frequencies in one-time-marching run.

The code we used in this paper is called XFDTD 7.0. Simulation model and field snapshot of EM propagation are shown in Figure 5. In this simulation, a human model is placed in free space. Relative permittivity and conductivity of the model are 50 and $1 \mathrm{~s} / \mathrm{m}$, respectively. An incident plane wave propagates at $0^{\circ}$ elevation with two polarization modes, vertical and horizontal, respectively. The incident plane wave is excitated by a Gauss impulse signal, which is shown at the left-top of Figure 5(a). 41 receivers are placed $2 \mathrm{~m}$ behind the human to receive the E-field. The total length and space length of receivers are $4 \mathrm{~m}$ and $0.1 \mathrm{~m}$, respectively. Figure 5(b) is a field snapshot of EM propagation in the simulation. Incident plane wave, incident direction, human scattering, and human obscuring are marked in the figure. In order to analyze human obscuring quantitatively, received signals are discussed in Figure 6.

Figures 6(a) and 6(b) show the electronic field strength for all the receiver sensors in $\mathrm{V}-\mathrm{V}$ polarization and $\mathrm{H}-\mathrm{H}$ polarization, respectively. The electronic field strength in full frequency range (up to $3.3 \mathrm{GHz}$ ) is shown in the left of each figure. Additionally, in the right of each figure, there are three subplots of electronic field strength at three separated frequencies: $1 \mathrm{GHz}, 2 \mathrm{GHz}$, and $3 \mathrm{GHz}$. It is noted that, on the one hand, human scatting is not isotropic. The electronic field strength varies significantly at different receiver positions, especially where the receiver is totally obscured by the human. On the other hand, as an extended target, 

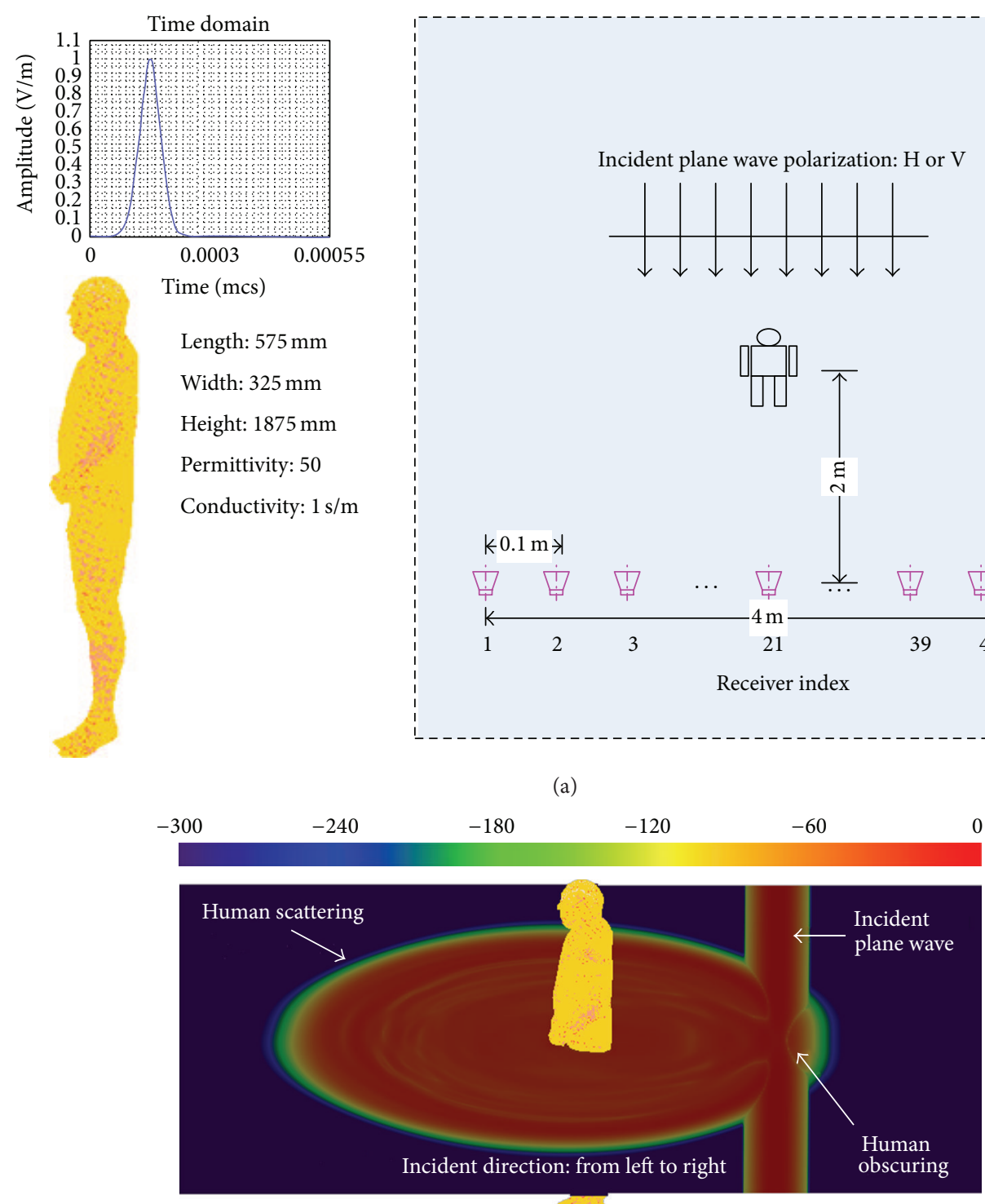

(b)

FIGURE 5: Simulation model and field snapshot in FDTD calculation. (a) Left-top: Gauss impulse signal is used for exciting the wave; leftdown: description about human model; right: simulation model in calculation. (b) Human obscuring effect in the field snapshot.

the human obscures EM propagation. As a result, electronic field strengths corresponding from the 17 th to the $23 \mathrm{rd}$ receivers are relatively small compared to other receivers. Besides, with frequency enlargement (larger than $1 \mathrm{GHz}$ in vertical polarization and $1.2 \mathrm{GHz}$ in horizontal polarization, resp.), the diffraction effect can be negligible. In this case, human obscuring is significantly severe.

(B) Experiments in MIMO Mode. Experiments about moving target imaging in MIMO mode have been done in National University of Defense Technology (NUDT). NUDT has developed a UWB MIMO through-the-wall radar system [31]. The radar system consists of separate transmit and receive antenna arrays. The receive array consists of fifteen elements with interelement spacing of $0.25 \mathrm{~m}$. The transmit array consists of two antennas, located at either end of the receive array. The waveform used in this system is stepped frequency signal, with frequency range from $500 \mathrm{MHz}$ to $2.5 \mathrm{GHz}$. The principle diagram of radar system is given in Figure 7.

Imaging data were collected by the radar system. The experimental imaging geometry is shown in Figure 8. Radar is placed at the left side of a cinderblock building, and the antenna array is parallel to the side wall, at a distance of $8 \mathrm{~m}$. The thickness of the cinderblock wall is $30 \mathrm{~cm}$. The width of the side wall is $9.3 \mathrm{~m}$. On the left side of the imaging scene there are some large trees. The experimental scene and the configuration inside the building are shown in Figure 8. 

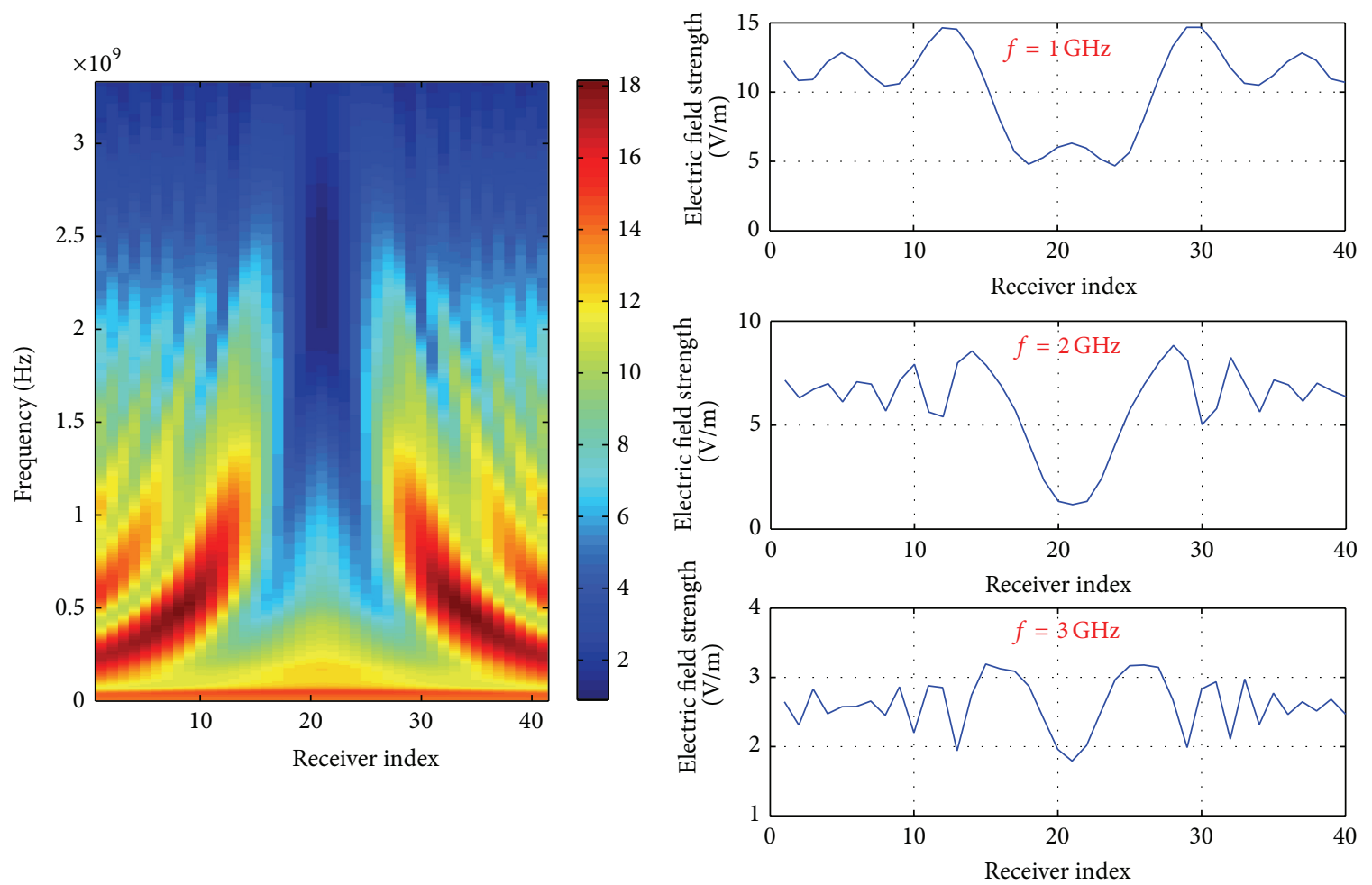

(a)
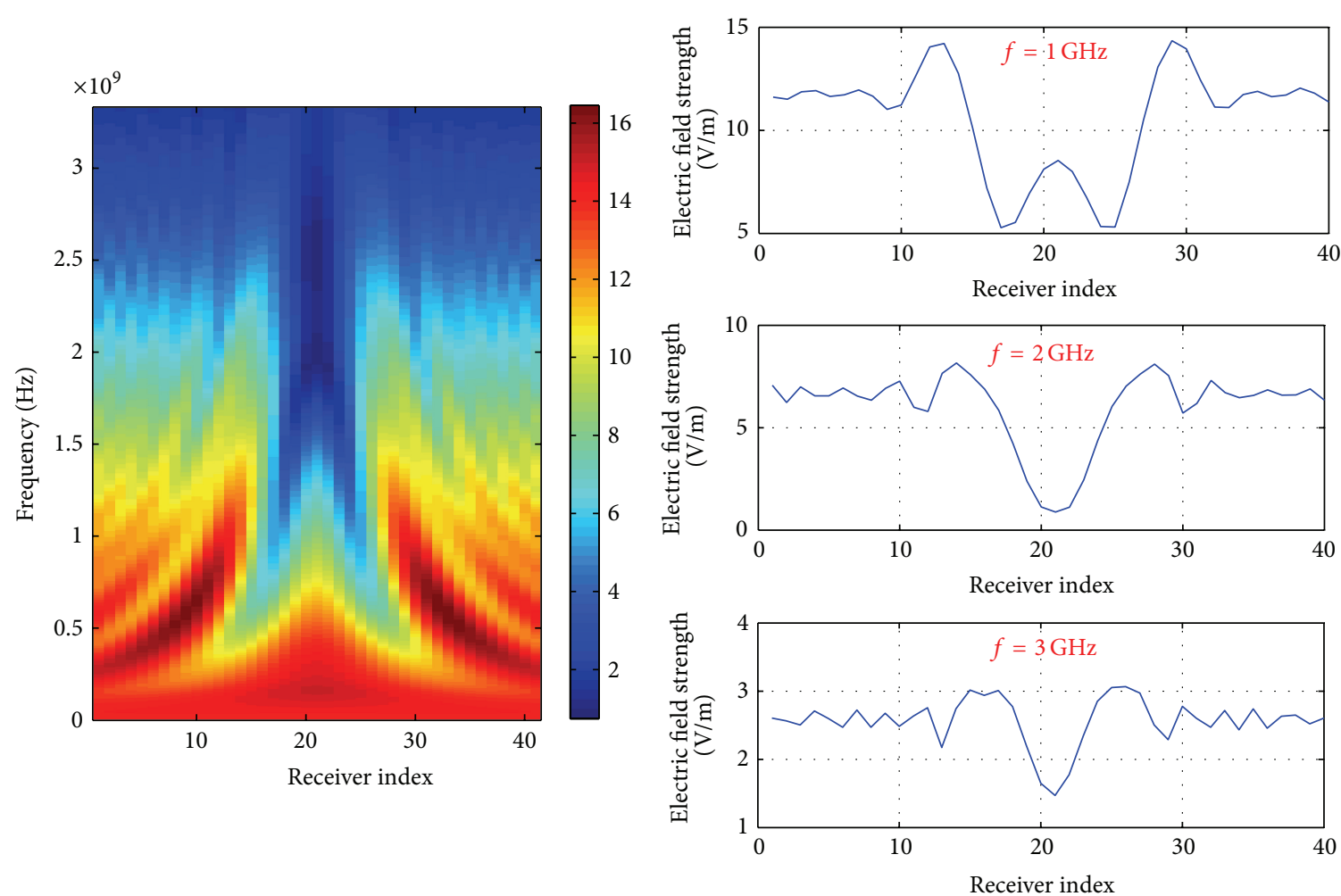

(b)

Figure 6: There are two subplots in each figure. Left: received electronic field strength for all the receivers with frequency up to $3.3 \mathrm{GHz}$; right: electronic field strength for all the receivers at three separated frequencies: $1 \mathrm{GHz}, 2 \mathrm{GHz}$, and $3 \mathrm{GHz}$. (a) V-V polarization. (b) H-H polarization. 


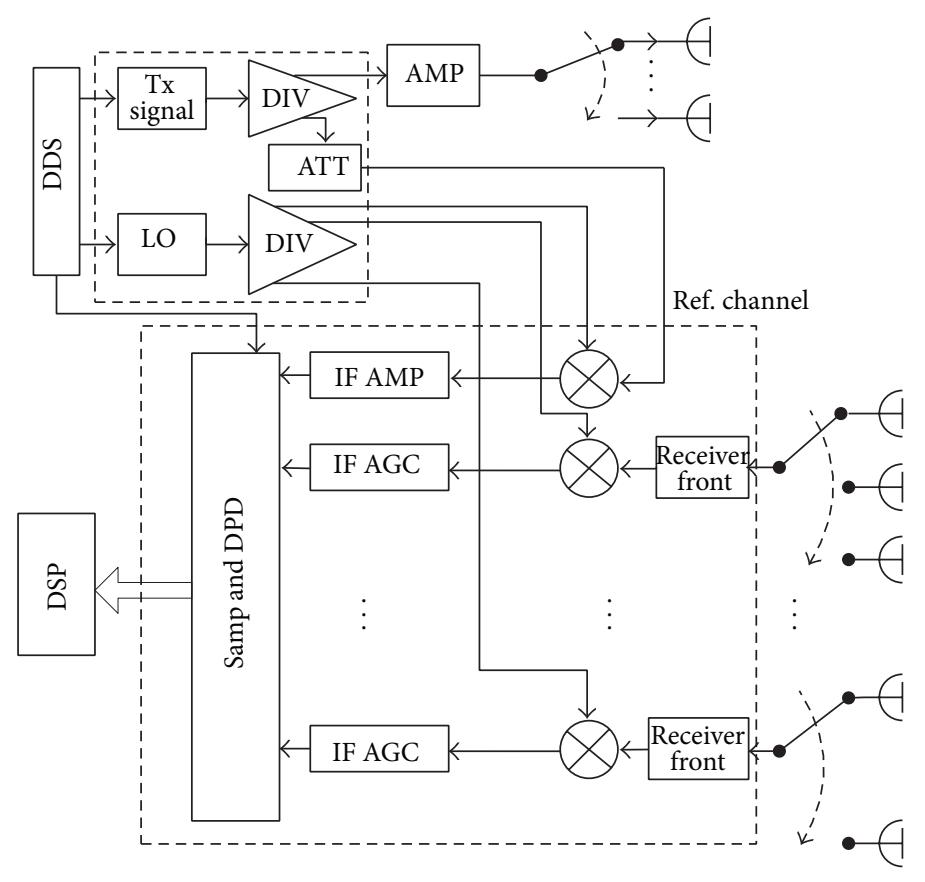

FIGURE 7: The principle diagram of through-the-wall radar system.

There are two experiments about moving target imaging. In the first experiment, one person walked in the bedroom $2 \mathrm{~m}$ behind the wall during data collection. Length of his track is $3 \mathrm{~m}$ (shown in Figure 9). The original imaging result after change detection is obtained via back projection (BP) imaging method. In the imaging, the frequency is from $1 \mathrm{GHz}$ to $2 \mathrm{GHz}$, with $2 \mathrm{MHz}$ increment. Taking the 27 th imaging result, for example, there are three targets shown in the figure, including one true target and two ghosts (shown in Figure 10). In the figure, P1 is the true target, while P2 and $\mathrm{P} 3$ are the ghosts corresponding the left transmitter and right transmitter, respectively.

The result after CFAR detection is shown in Figure 11. The true target (P1) as well as two ghosts (P2, P3) has been detected in the figure. The energy of ghost P3 is even larger than true target P1. The ghosts severely affect the accuracy of detection.

Using the proposed method in this paper, the corresponding result is obtained in Figure 12. Compared with Figure 10, it is shown that ghosts have been removed efficiently. Besides, the clutter has also been suppressed via the proposed method.

In the second experiment, two humans walked along the diagonal line in a bedroom during data collection (shown in Figure 13). In this experiment, imaging parameters and imaging method are the same as the first one. Taking the 30th imaging result, for example, there are six targets in the figure, including two true targets and four ghosts (shown in Figure 14). In Figure 14, P1 and P2 are true targets. P3 and P4 are ghosts, which are caused by P1 corresponding to the right transmitter and the left transmitter, respectively. P5 and P6 are ghosts, which are caused by P2 corresponding to the right transmitter and the left transmitter, respectively. Besides the ghost problem, strong clutter also affects imaging quality severely.

Figure 15 is the imaging result after CFAR detection. In Figure 15, two true targets (P1 and P2) are detected. However, two ghosts (P3, P4) are also present. Adopting the proposed method in this paper, we obtain the processing result in Figure 16. It is noted that all the ghosts are efficiently removed and the imaging quality is also significantly improved.

(C) Electromagnetic Simulation in Monostatic Mode. To validate the performance of the proposed method in monostatic through-the-wall mode, we construct an electromagnetic simulation via XFDTD code. As shown in Figure 17, one human moves in a four-wall room. The length and width of the building are 6 and $5 \mathrm{~m}$, respectively. The thickness of the wall is $0.3 \mathrm{~m}$, and its relative permittivity is 4.2 . The dimensions of the human model are $575 \times 325 \times 1875 \mathrm{~mm}$. Its relative permittivity and conductivity are 50 and $1 \mathrm{~s} / \mathrm{m}$, respectively. There are 63 antennas in front of the wall to transmit and receive signals in monostatic mode. A $3 \mathrm{GHz}$ band impulse signal is adopted in this simulation.

Resorting to the Fourier transformation and interpolation method, the frequency used in the imaging ranges from 0.5 to $1 \mathrm{GHz}$ with $2 \mathrm{MHz}$ steps. Taking the 15th imaging results, for example, Figure 18 is the original imaging result. In this figure, P1 is the true target and P2 is the ghost. By using the CFAR detection, the true target P1 is detected but the ghost P2 is also present (shown in Figure 19). Adopting the proposed method in this paper, the processing result is given in Figure 20. Compared with Figures 18 and 19, it is shown that the ghosts are well mitigated and the target has a correspondingly higher image quality. 


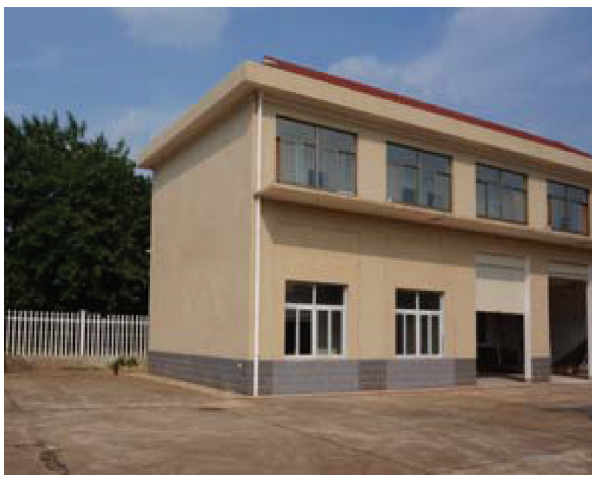

(a)

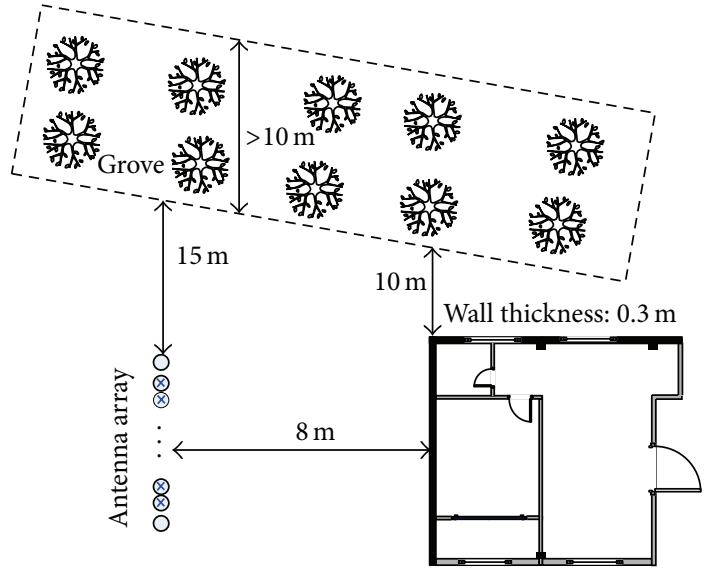

O Transmit antenna

$\otimes$ Receive antenna

(b)

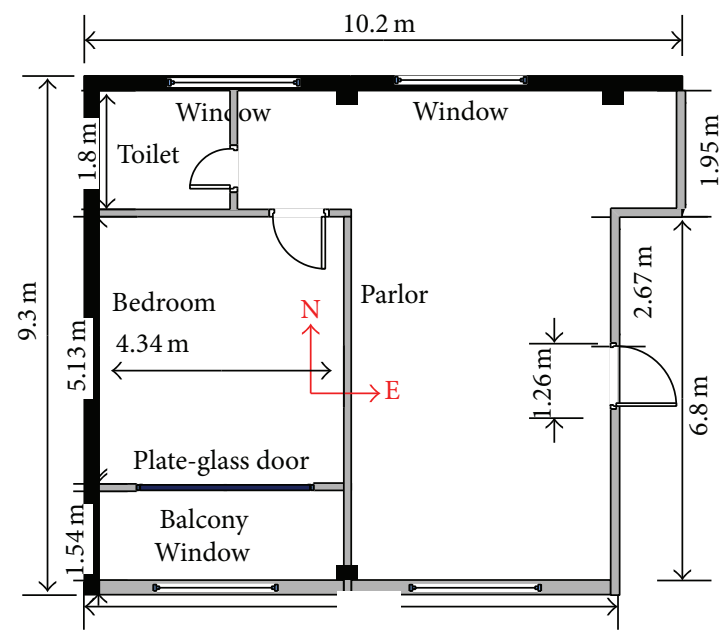

(c)

FIGURE 8: Experimental imaging geometry and scene. (a) Photograph of the building. (b) Geometry of antenna array with the cinderblock building. (c) Configuration inside the building.

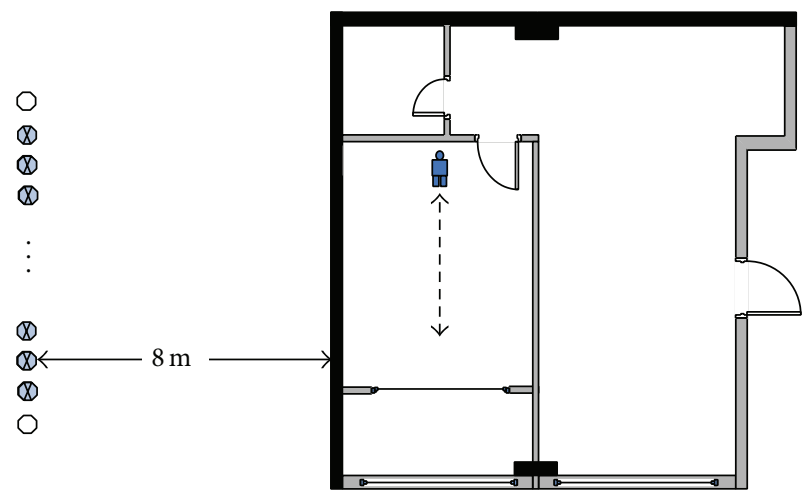

(a)

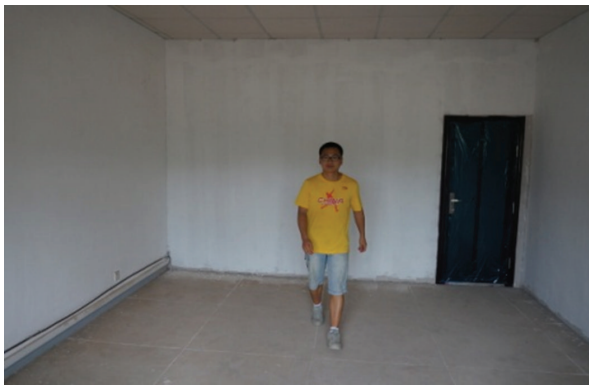

(b)

Figure 9: One person walked in the bedroom. (a) Track of the movement. (b) Photo of the scene when one person was walking $2 \mathrm{~m}$ behind the wall in bedroom. 


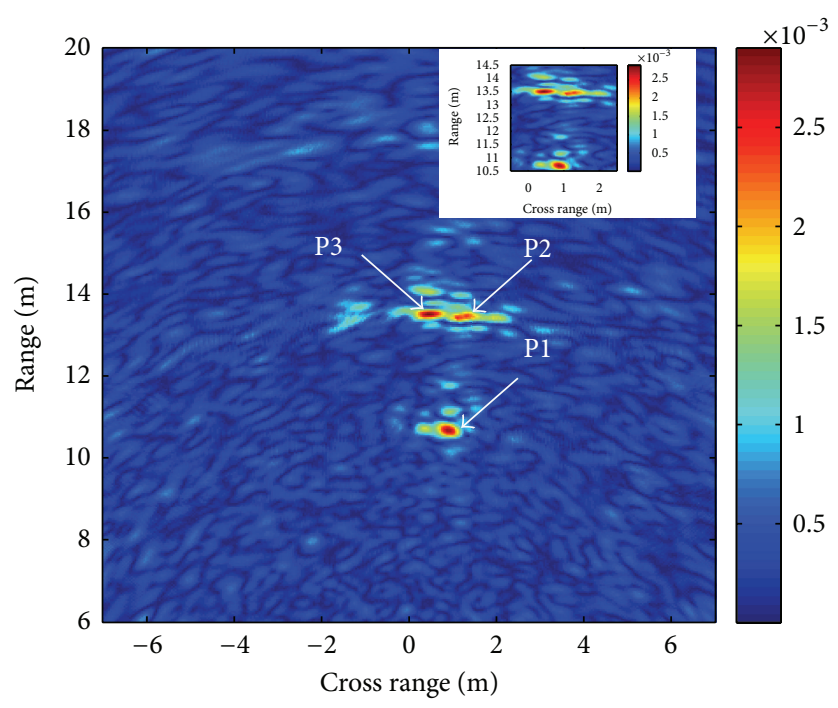

FIGURE 10: The original moving target imaging result.

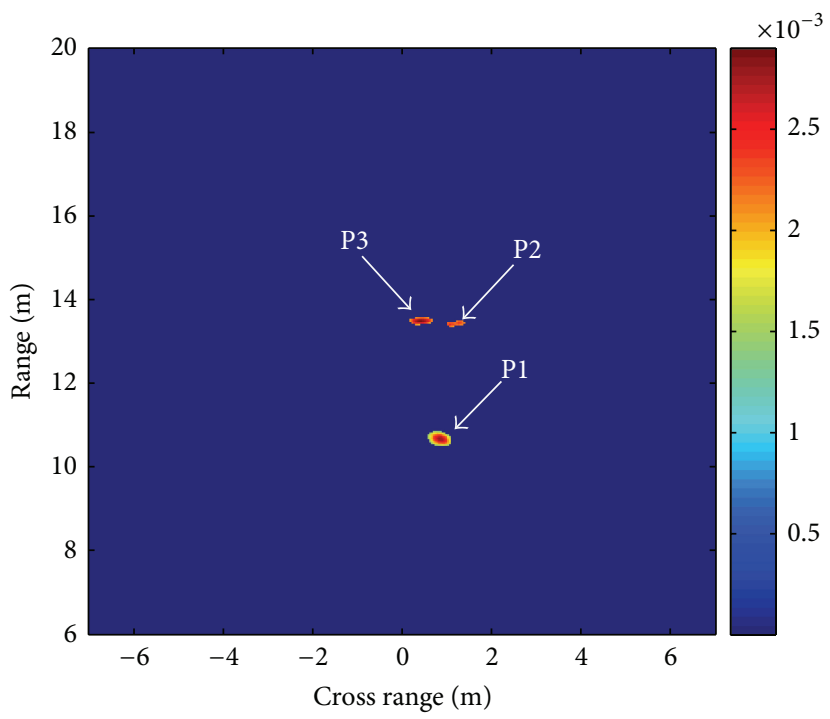

FIGURE 11: After CFAR detection of Figure 10, ghosts are detected in the figure.

\section{Conclusion}

In high-resolution TWI application, considering the relationship between target size and resolution, most of targets such as human and furniture are extended targets rather than point targets. Therefore, the previous TWI signal model which is based on point target assumption cannot accurately describe the real characteristics of EM propagation. In this paper, by using the extended target theory, two new TWI signal models for extended target, in MIMO and the monostatic modes, are constructed. The expressions of wall reflection and target scattering are derived.

Compared with the conventional point target signal models, a new kind of ghost problem is discovered. It is caused by target obscuring in EM propagation. This problem brings severe effect on the imaging, especially on moving target imaging. Using the proposed models, we exploited the mechanism of the problem and analyzed the characteristics of the ghost.

To improve the image quality in moving target imaging, an efficient approach which adopts CFAR, clustering method, and spatial geometry relationship is proposed to remove the ghosts. By using the synthetic and experimental data, the proposed models are shown in accordance with the processing results. Besides, the efficiency of the proposed method is also validated, which shows that the ghosts are efficiently mitigated and the image quality is significantly improved.

It is mentioned that, in this paper, all the processing is applied based on the original imaging results. Because of the refraction phenomenon, the obscured area is not strictly on a line with the antenna (or mirror antenna) and 


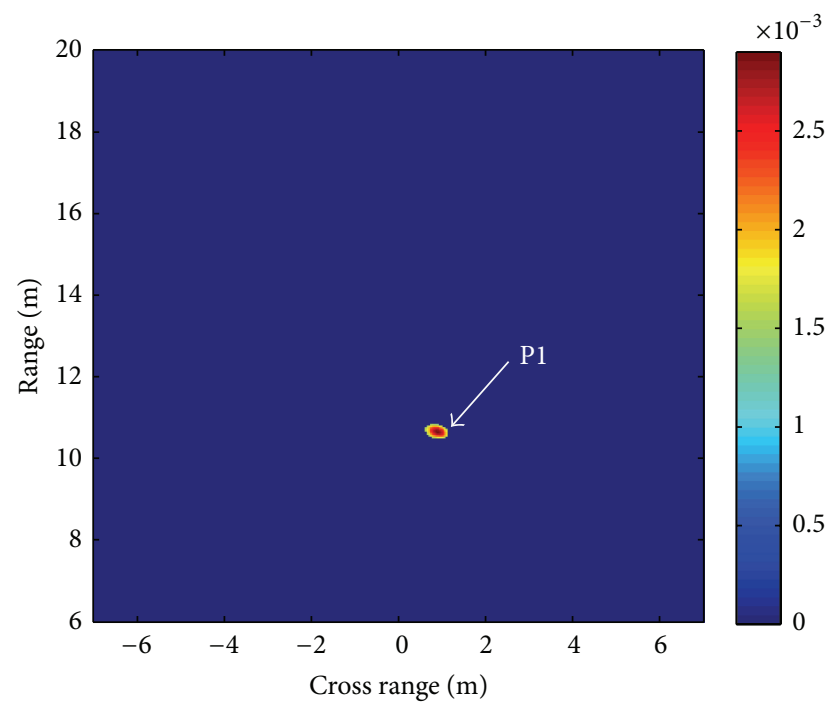

FIGURE 12: Imaging result after processing by the proposed method in this paper.

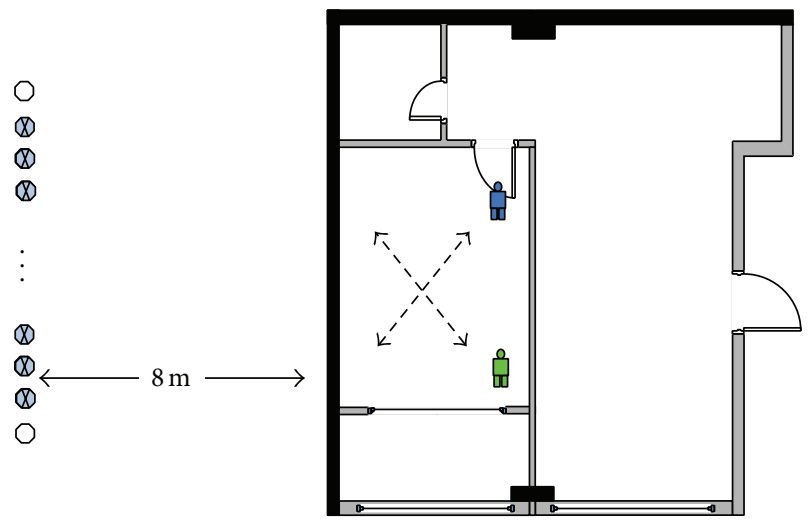

(a)

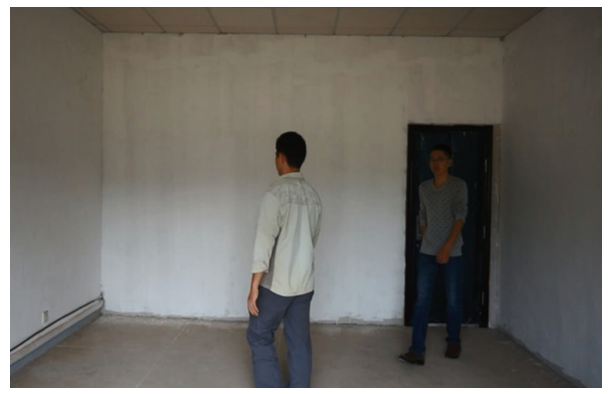

(b)

FIGURE 13: Two persons walked in the bedroom. (a) Track of the movement. (b) Photo of scene when two persons were walking in the bedroom.

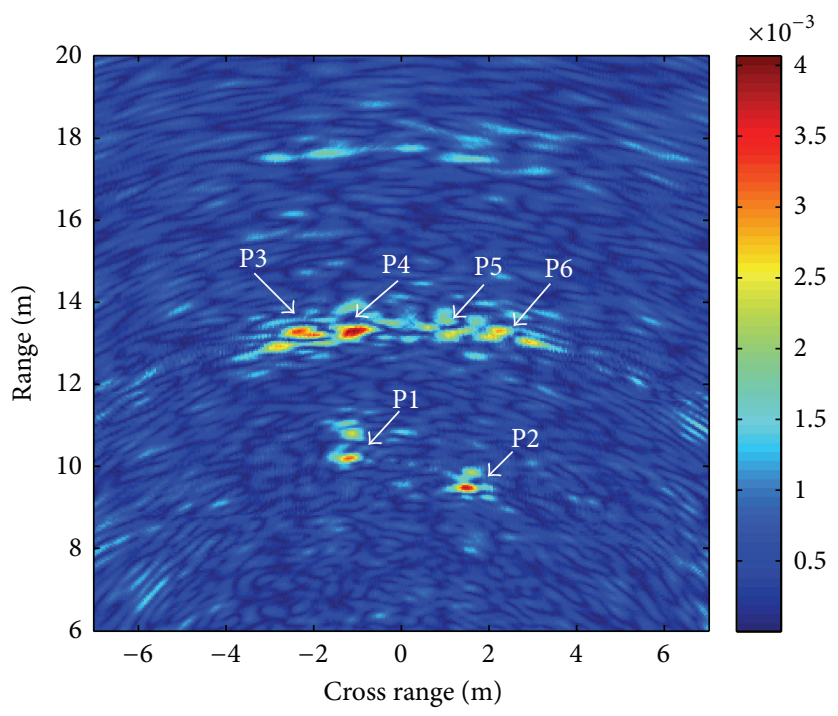

FIGURE 14: The original moving target imaging result. There are six targets in the image, including two true targets and four ghosts. 


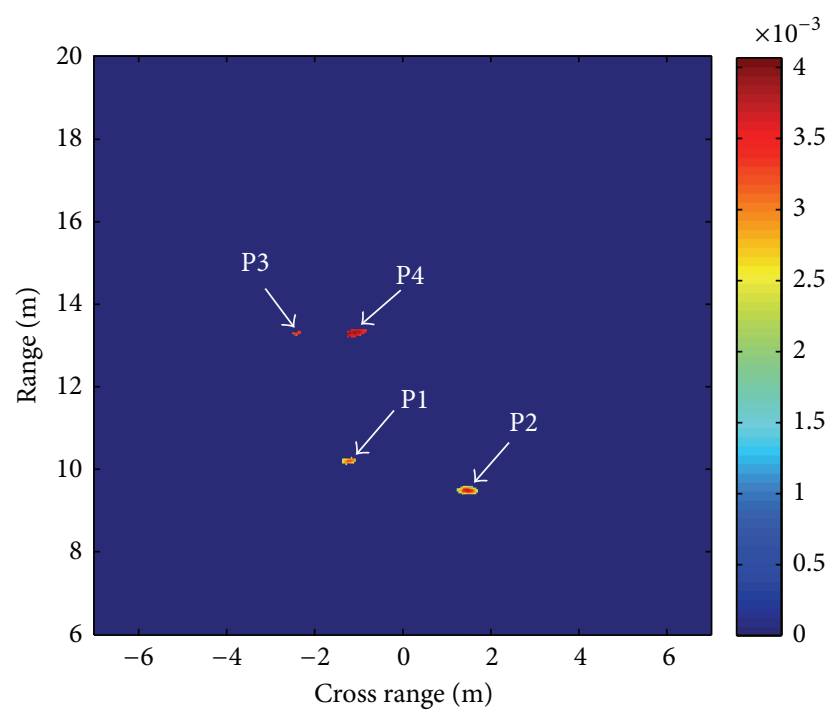

FIGURE 15: Two true targets as well as three ghosts have been detected in the image after CFAR processing.

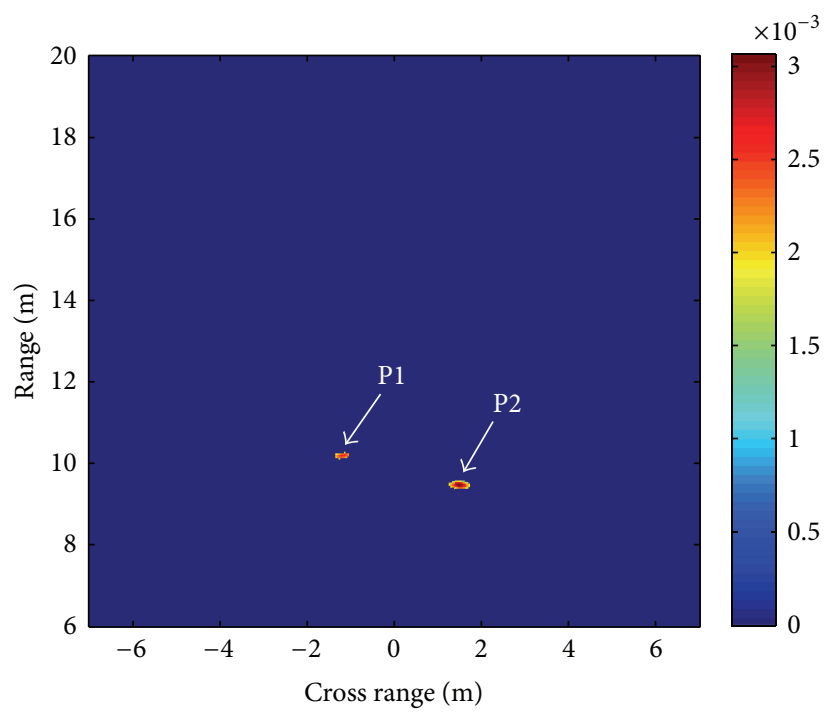

FIGURE 16: Imaging result processed by the proposed method in this paper.

the target in MIMO mode. However, in the original image, the imaged positions of the targets and ghosts are behind their true positions, that is, shifting $d(\sqrt{\varepsilon}-1)$ behind their true positions. Therefore, the refraction brings the errors, while the conventional BP image makes the imaged position errors. As a result, the influence of the two errors may cancel out, thus achieving a relatively satisfactory ghost-mitigated image by the proposed method.

\section{Appendix}

Figure 21 shows the signal channel and the obscured area in MIMO mode. Figure 17(a) corresponds to the first case in which the obscuring occurs before the back wall reflection. Figure 17(b) demonstrates the obscuring in the second case; that is, the obscuring occurs after the back wall reflection.

In Figure 21, a target is placed in a four-wall room. The target center is located at $\left(x_{p}, y_{p}\right) . H$ and $H^{\prime}$ denote the left end and right end of the target. $P_{H} P_{H}^{\prime}$ and $Q_{H} Q_{H}^{\prime}$ denote the obscured area in the first answer second case, respectively. For convenience, in following derivation, we define the positions of $T_{1}, A_{1}, A_{1}^{\prime}, H, H^{\prime}, P_{H}, P_{H}^{\prime}, Q_{H}$, and $Q_{H}^{\prime}$ as $\left(x_{T_{1}}, y_{T_{1}}\right),\left(x_{A_{1}}, y_{A_{1}}\right),\left(x_{A_{1}^{\prime}}, y_{A_{1}^{\prime}}\right),\left(x_{H}, y_{H}\right),\left(x_{H^{\prime}}, y_{H^{\prime}}\right)$, $\left(x_{P_{H}}, y_{P_{H}}\right),\left(x_{P_{H}^{\prime}}, y_{P_{H}^{\prime}}\right),\left(x_{Q_{H}}, y_{Q_{H}}\right)$, and $\left(x_{Q_{H}^{\prime}}, y_{Q_{H}^{\prime}}\right)$, respectively. For convenience, only the obscured areas for antenna $T_{1}$ are derived as an example in this appendix. 


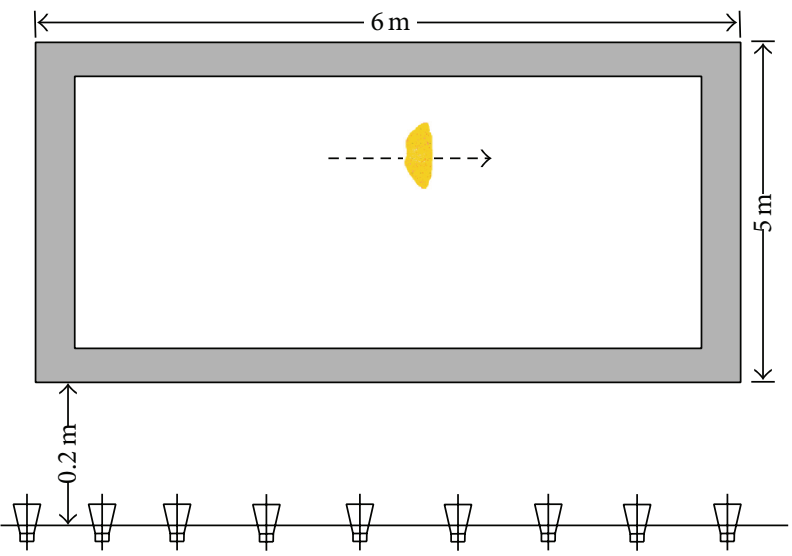

(a)
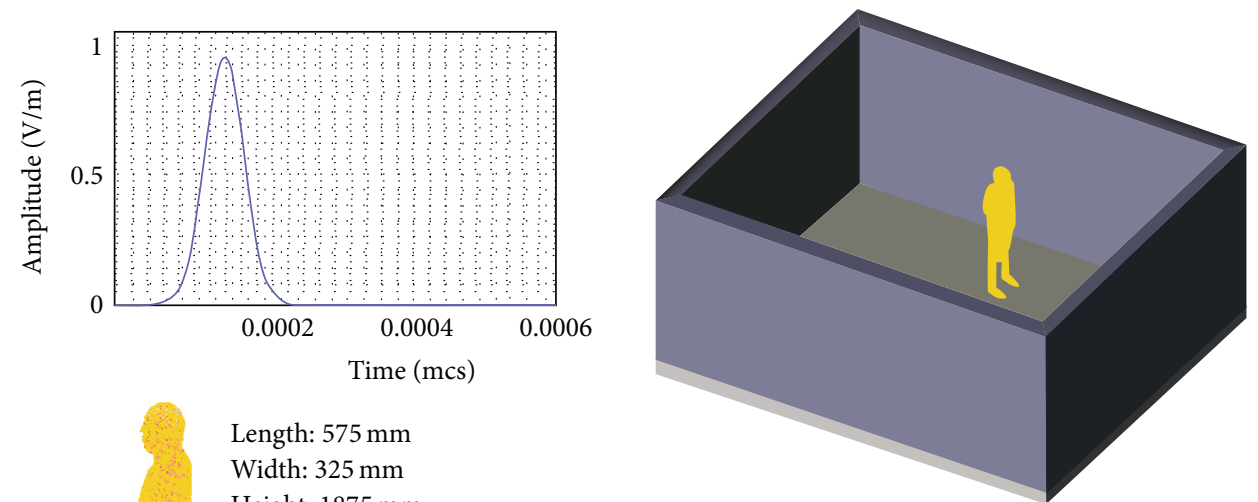

Length: $575 \mathrm{~mm}$

Width: $325 \mathrm{~mm}$

Height: $1875 \mathrm{~mm}$

Permittivity: 50

Conductivity: $1 \mathrm{~s} / \mathrm{m}$

(b)

FIGURE 17: Simulation scene and 3D model. (a) A human moves in a four-wall room in monostatic through-the-wall mode. (b) Top: 3 GHz band impulse signal in the time domain; left-bottom: the human model used in the simulation; right-bottom: 3D model of the scene.

In Figure 21(a), according to the geometry relationship and projection approximation, we can obtain

$$
\begin{gathered}
\frac{\mathrm{OT}-X-Y}{d_{3}}=\frac{X}{d_{1}}, \\
\mathrm{OT}=x_{H}-x_{T_{1}}, \\
\frac{d}{Y} \cdot \frac{\mathrm{OT}-X}{d+d_{3}}=\sqrt{\varepsilon} .
\end{gathered}
$$

By solving the above equation, we obtain

$$
\begin{aligned}
& X=\frac{d_{1}\left(x_{H}-x_{T_{1}}\right)\left[\sqrt{\varepsilon}\left(d_{3}+d\right)-d\right]}{\sqrt{\varepsilon}\left(d_{1}+d_{3}\right)\left(d_{3}+d\right)-d d_{1}}, \\
& Y=\frac{d_{3} d\left(x_{H}-x_{T_{1}}\right)}{\sqrt{\varepsilon}\left(d_{1}+d_{3}\right)\left(d_{3}+d\right)-d d_{1}} .
\end{aligned}
$$

So the positions of the refraction points $A_{1}$ and $A_{1}^{\prime}$ can be obtained:

$$
\begin{gathered}
\mathbf{x}_{A_{1}}=\left(x_{T_{1}}+\frac{\left(x_{H}-x_{T_{1}}\right) \cdot d_{1}\left[\sqrt{\varepsilon}\left(d_{3}+d\right)-d\right]}{\sqrt{\varepsilon}\left(d_{1}+d_{3}\right)\left(d_{3}+d\right)-d d_{1}} y_{T_{1}}+d_{1}\right), \\
\mathbf{x}_{A_{1}^{\prime}}=\left(x_{T_{1}}+\frac{\left(x_{H}-x_{T_{1}}\right) \cdot d_{1}\left[\sqrt{\varepsilon}\left(d_{3}+d\right)-d\right]}{\sqrt{\varepsilon}\left(d_{1}+d_{3}\right)\left(d_{3}+d\right)-d d_{1}}+\frac{\left(x_{H}-x_{T_{1}}\right) \cdot d_{3} \cdot d}{\sqrt{\varepsilon}\left(d_{1}+d_{3}\right)\left(d_{3}+d\right)-d d_{1}} y_{T_{1}}+d_{1}+d\right) .
\end{gathered}
$$




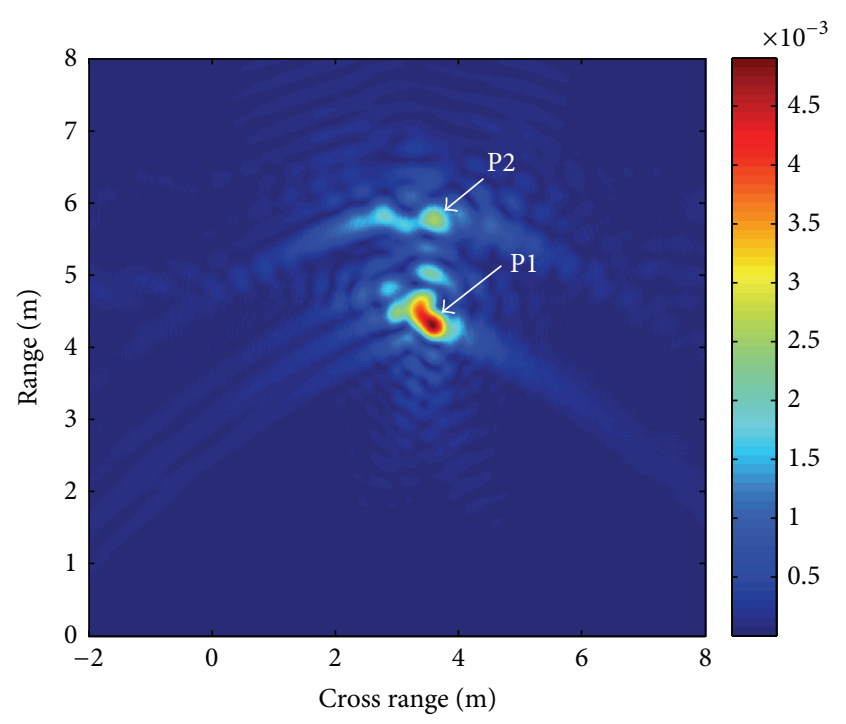

FIGURE 18: The original moving target imaging result in the monostatic mode.

According to the geometry relationship, the length from point $M$ to $P_{H}$ is

$$
M P_{H}=\left(y_{T_{1}}+d_{1}+d+d_{2}-y_{H}\right) \cdot \frac{\mathrm{OT}-X-Y}{y_{H}-y_{T_{1}}-d_{1}-d} .
$$

Then, the position of $P_{H}$ can be computed. It is

$$
\begin{aligned}
& x_{P_{H}}=x_{H}+\left(\left(x_{H}-x_{T_{1}}\right)\left(\sqrt{\varepsilon} y_{H}-\sqrt{\varepsilon} y_{T_{1}}-\sqrt{\varepsilon} d-d\right)\right. \\
&\left.\times\left(y_{T_{1}}+d_{1}+d+d_{2}-y_{H}\right)\right) \\
& \times\left(\sqrt{\varepsilon}\left(y_{H}-y_{T_{1}}-d\right)\left(y_{H}-y_{T_{1}}-d_{1}\right)-d d_{1}\right)^{-1}, \\
& x_{H}>x_{T_{1}} .
\end{aligned}
$$

Here,

$$
x_{H}=x_{p}-\frac{L}{2}, \quad y_{H}=y_{p} .
$$

So (A.5) is rewritten as

$$
\begin{aligned}
x_{P_{H}}= & x_{p}-\frac{L}{2} \\
+ & \left(\left(x_{p}-\frac{L}{2}-x_{T_{1}}\right)\left(\sqrt{\varepsilon} y_{p}-\sqrt{\varepsilon} y_{T_{1}}-\sqrt{\varepsilon} d-d\right)\right. \\
& \left.\times\left(y_{T_{1}}+d_{1}+d+d_{2}-y_{p}\right)\right) \\
& \times\left(\sqrt{\varepsilon}\left(y_{p}-y_{T_{1}}-d\right)\left(y_{p}-y_{T_{1}}-d_{1}\right)-d d_{1}\right)^{-1}, \\
& x_{p}>x_{T_{1}}+\frac{L}{2} .
\end{aligned}
$$

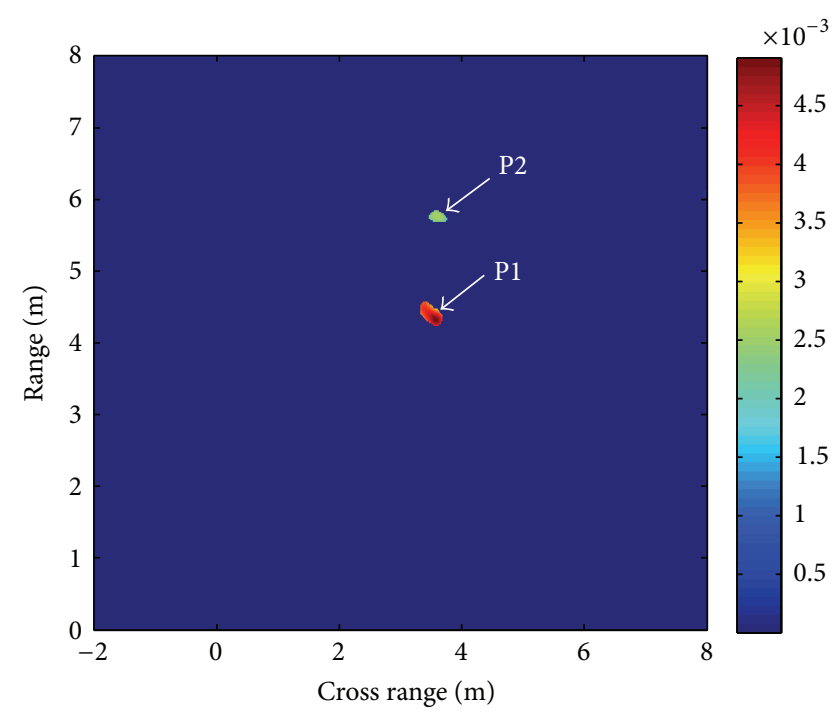

FIGURE 19: The true target and the ghost have been detected in the image after CFAR processing.

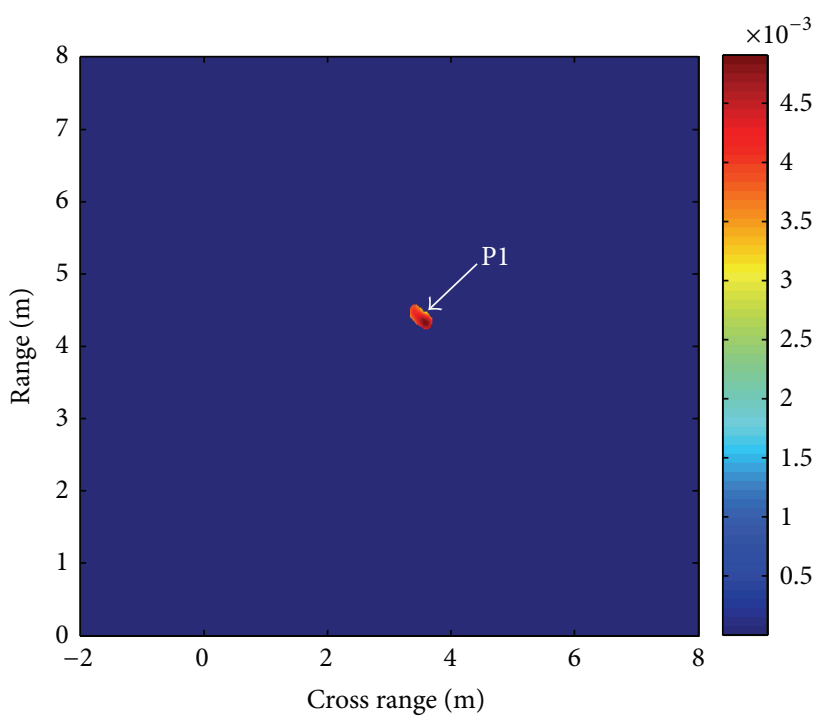

FIGURE 20: The ghost is mitigated by the proposed method in this paper.

Anonymously, the position of $P_{H}^{\prime}$ is

$$
\begin{aligned}
& x_{P_{H}^{\prime}}=x_{p}+\frac{L}{2} \\
&+\left(\left(x_{p}+\frac{L}{2}-x_{T_{1}}\right)\left(\sqrt{\varepsilon} y_{p}-\sqrt{\varepsilon} y_{T_{1}}-\sqrt{\varepsilon} d-d\right)\right. \\
& \quad\left.\times\left(y_{T_{1}}+d_{1}+d+d_{2}-y_{p}\right)\right) \\
& \times\left(\sqrt{\varepsilon}\left(y_{p}-y_{T_{1}}-d\right)\left(y_{p}-y_{T_{1}}-d_{1}\right)-d d_{1}\right)^{-1}, \\
& x_{p}>x_{T_{1}}+\frac{L}{2} .
\end{aligned}
$$




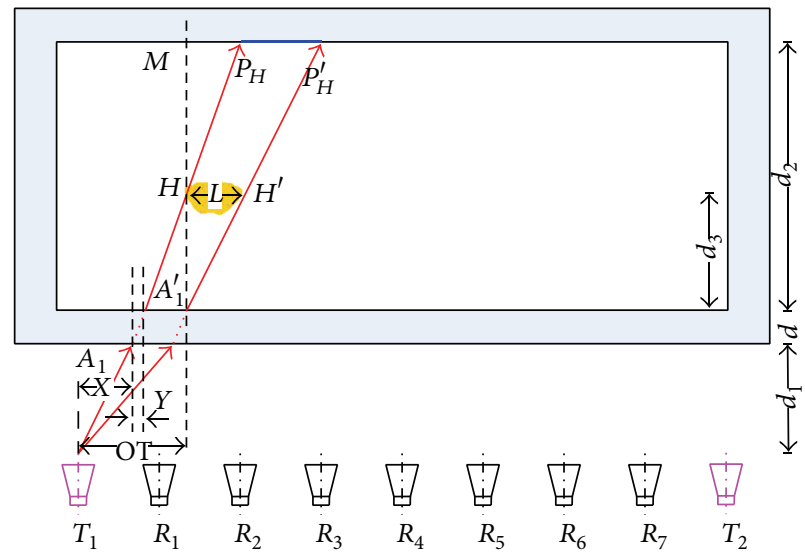

(a)

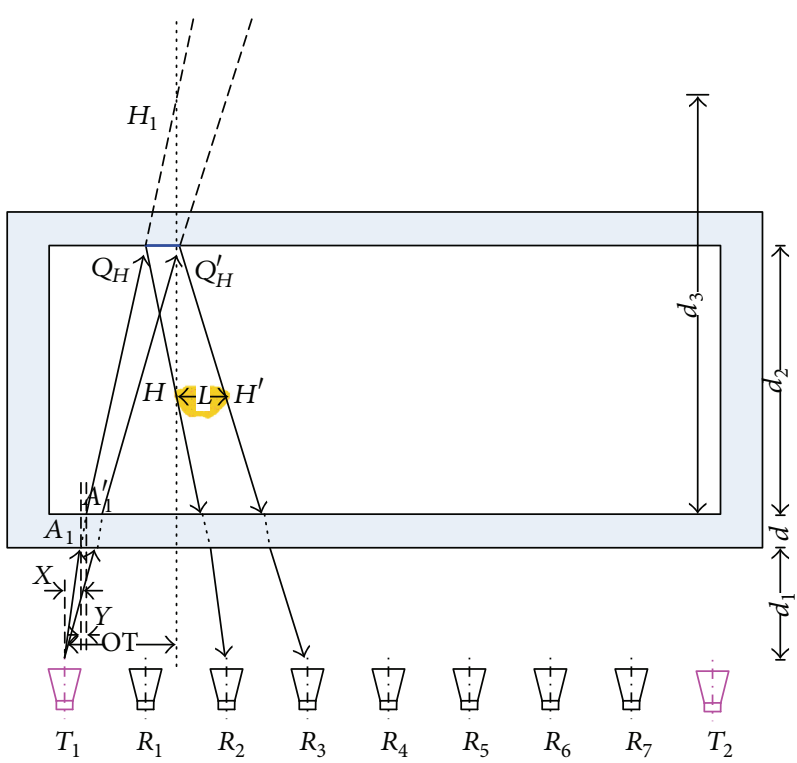

(b)

FIgURE 21: Target obscures the EM propagation in MIMO mode. (a) The first case: obscuring occurs before the back wall reflection. (b) The second case: obscuring occurs after the back wall reflection.

Therefore, the obscured area in the first case is

$$
\Pi_{11}=\left\{\begin{array}{l}
x_{\mathrm{ob}} \in\left[\begin{array}{ll}
x_{P_{H}} & x_{P_{H}^{\prime}}
\end{array}\right] \\
y_{\mathrm{ob}}=d_{1}+d+d_{2}+y_{T_{1}}
\end{array} \quad x_{p}>x_{T_{1}}+\frac{L}{2} .\right.
$$

Figure 21(b) shows the second case in which the obscuring occurs after the back wall reflection. To compute the positions of $Q_{H}$ and $Q_{H}^{\prime}$, we mark the mirror point of $H$; that is, $H_{1}=\left(x_{H_{1}}, y_{H_{1}}\right)$. Here,

$$
x_{H_{1}}=x_{H}, \quad y_{H_{1}}=y_{T_{1}}+2\left(d_{1}+d+d_{2}\right)-y_{H} .
$$

Then, according to the geometry relationship and projection approximation, we can obtain

$$
\begin{gathered}
\frac{\mathrm{OT}-X-Y}{d_{3}}=\frac{X}{d_{1}}, \\
\mathrm{OT}=x_{H}-x_{T_{1}}, \\
\frac{d}{Y} \cdot \frac{\mathrm{OT}-X}{d+d_{3}}=\sqrt{\varepsilon} .
\end{gathered}
$$

By solving the above equation, the positions of the refraction points $A_{1}$ and $A_{1}^{\prime}$ can be obtained:

$$
\begin{gathered}
\mathbf{x}_{A_{1}}=\left(x_{T_{1}}+\frac{\left(x_{H}-x_{T_{1}}\right) \cdot d_{1}\left[\sqrt{\varepsilon}\left(d_{1}+2 d+2 d_{2}-y_{H}\right)-d\right]}{\sqrt{\varepsilon}\left(2 d_{1}+d+2 d_{2}-y_{H}\right)\left(d_{1}+2 d+2 d_{2}-y_{H}\right)-d d_{1}} y_{T_{1}}+d_{1}\right), \quad x_{H}>x_{T_{1}}, \\
\mathbf{x}_{A_{1}^{\prime}}=\left(x_{T_{1}}+\frac{\left(x_{H}-x_{T_{1}}\right) \cdot d_{1}\left[\sqrt{\varepsilon}\left(d_{1}+2 d+2 d_{2}-y_{H}\right)-d\right]+\left(x_{H}-x_{T_{1}}\right) \cdot\left(d_{1}+d+2 d_{2}-y_{H}\right) \cdot d}{\sqrt{\varepsilon}\left(2 d_{1}+d+2 d_{2}-y_{H}\right)\left(d_{1}+2 d+2 d_{2}-y_{H}\right)-d d_{1}} y_{T_{1}}+d_{1}+d\right),
\end{gathered}
$$

According to geometry relationship, we have

$$
\frac{x_{A_{1}^{\prime}}-x_{Q_{H}}}{y_{A_{1}^{\prime}}-y_{Q_{H}}}=\frac{x_{A_{1}^{\prime}}-x_{H_{1}}}{y_{A_{1}^{\prime}}-y_{H_{1}}} .
$$

Here,

$$
y_{Q_{H}}=y_{T_{1}}+d_{1}+d+d_{2} .
$$

Therefore, we obtain

$$
\begin{aligned}
x_{\mathrm{Q}_{H}}=x_{A_{1}^{\prime}}-\frac{x_{A_{1}^{\prime}}-x_{H_{1}}}{y_{A_{1}^{\prime}}-y_{H_{1}}}\left(y_{A_{1}^{\prime}}-y_{\mathrm{Q}_{H}}\right) \\
=\frac{\left(y_{H}-d_{1}-d-d_{2}\right) x_{A_{1}^{\prime}}-d_{2} x_{H}}{y_{H}-d_{1}-d-2 d_{2}}
\end{aligned}
$$




$$
\begin{gathered}
=\frac{\left(y_{H}-d_{1}-d-d_{2}\right) x_{T_{1}}-d_{2} x_{H}}{y_{H}-d_{1}-d-2 d_{2}} \\
+\left(\left(x_{H}-x_{T_{1}}\right) \cdot\left(y_{H}-d_{1}-d-d_{2}\right)\right. \\
\cdot\left[\left(d_{1}^{2}+2 d d_{1}+2 d_{2} d_{1}\right) \sqrt{\varepsilon}+d^{2}\right. \\
\left.\left.\quad+2 d_{2} d-\left(d_{1} \sqrt{\varepsilon}+d\right) y_{H}\right]\right) \\
\times\left(\left[\sqrt{\varepsilon}\left(2 d_{1}+d+2 d_{2}-y_{H}\right)\right.\right. \\
\left.\times\left(d_{1}+2 d+2 d_{2}-y_{H}\right)-d d_{1}\right] \\
\left.\times\left(y_{H}-d_{1}-d-2 d_{2}\right)\right)^{-1}, \quad x_{H}>x_{T_{1}} .
\end{gathered}
$$

Here,

$$
x_{H}=x_{p}-\frac{L}{2}, \quad y_{H}=y_{p}
$$

So (A.15) is rewritten as

$$
\begin{aligned}
x_{\mathrm{Q}_{H}}= & \left(\left(y_{p}-d_{1}-d-d_{2}\right) x_{T_{1}}-d_{2}\left(x_{p}-\frac{L}{2}\right)\right) \\
& \times\left(y_{p}-d_{1}-d-2 d_{2}\right)^{-1} \\
& +\left(\left(x_{p}-\frac{L}{2}-x_{T_{1}}\right) \cdot\left(y_{p}-d_{1}-d-d_{2}\right)\right. \\
& \quad \cdot\left[\left(d_{1}^{2}+2 d d_{1}+2 d_{2} d_{1}\right) \sqrt{\varepsilon}\right. \\
& \left.\left.\quad+d^{2}+2 d_{2} d-\left(d_{1} \sqrt{\varepsilon}+d\right) y_{p}\right]\right) \\
& \left.\quad \times\left(d_{1}+2 d+2 d_{2}-y_{p}\right)-d d_{1}\right] \\
& \left.\quad \times\left(y_{p}-d+d-d-2 d_{2}\right)\right)^{-1}, \quad x_{p}>x_{T_{1}}+\frac{L}{2} .
\end{aligned}
$$

Anonymously, the positions of the point $Q_{H}^{\prime}$ are

$$
\begin{aligned}
x_{\mathrm{Q}_{H}}= & \left(\left(y_{p}-d_{1}-d-d_{2}\right) x_{T_{1}}-d_{2}\left(x_{p}+\frac{L}{2}\right)\right) \\
& \times\left(y_{p}-d_{1}-d-2 d_{2}\right)^{-1} \\
& +\left(\left(x_{p}+\frac{L}{2}-x_{T_{1}}\right) \cdot\left(y_{p}-d_{1}-d-d_{2}\right)\right. \\
& \cdot\left[\left(d_{1}^{2}+2 d d_{1}+2 d_{2} d_{1}\right) \sqrt{\varepsilon}+d^{2}\right. \\
& \left.\left.+2 d_{2} d-\left(d_{1} \sqrt{\varepsilon}+d\right) y_{p}\right]\right) \\
& \times\left(\left[\sqrt{\varepsilon}\left(2 d_{1}+d+2 d_{2}-y_{p}\right)\right.\right.
\end{aligned}
$$

$$
\begin{aligned}
& \left.\times\left(d_{1}+2 d+2 d_{2}-y_{p}\right)-d d_{1}\right] \\
& \left.\times\left(y_{p}-d_{1}-d-2 d_{2}\right)\right)^{-1}, \quad x_{p}>x_{T_{1}}+\frac{L}{2} .
\end{aligned}
$$

Therefore, the obscured area in the second case is

$$
\Pi_{12}=\left\{\begin{array}{l}
x_{\mathrm{ob}} \in\left[\begin{array}{ll}
x_{\mathrm{Q}_{H}} & x_{\mathrm{Q}_{H}^{\prime}}
\end{array}\right] \\
y_{\mathrm{ob}}=d_{1}+d+d_{2}+y_{T_{1}},
\end{array} \quad x_{p}>x_{T_{1}}+\frac{L}{2} .\right.
$$

\section{Conflict of Interests}

The authors declare that there is no conflict of interests regarding the publication of this paper.

\section{Acknowledgment}

This work was supported in part by the National Natural Science Foundation of China under Grant nos. 61372161 and 61271441 .

\section{References}

[1] M. G. Amin, Through-the-Wall Radar Imaging, CRC Press, New York, NY, USA, 2010.

[2] G. E. Smith and B. G. Mobasseri, "Robust through-the-wall radar image classification using a target-model alignment procedure," IEEE Transactions on Image Processing, vol. 21, no. 2, pp. 754-767, 2012.

[3] V. H. Tang, A. Bouzerdoum, and S. L. Phung, "Two-stage through-the-wall radar image formation using compressive sensing," Journal of Electronic Imaging, vol. 22, no. 2, pp. 1-15, 2013.

[4] K. E. Browne, R. J. Burkholder, and J. L. Volakis, "Fast optimization of through-wall radar images via the method of lagrange multipliers," IEEE Transactions on Antennas and Propagation, vol. 61, no. 1, pp. 320-328, 2013.

[5] C. Debes, A. M. Zoubir, and M. G. Amin, "Enhanced detection using target polarization signatures in through-the-wall radar imaging," IEEE Transactions on Geoscience and Remote Sensing, vol. 50, no. 5, pp. 1968-1979, 2012.

[6] A. Fauzia and M. G. Amin, "Through-the-wall human motion indication using sparsity-driven change detection," IEEE Transactions on Geoscience and Remote Sensing, vol. 51, no. 2, pp. 881890, 2013.

[7] H. Estephan, M. G. Amin, and K. M. Yemelyanov, "Optimal waveform design for improved indoor target detection in sensing through-the-wall application," IEEE Transactions on Geoscience and Remote Sensing, vol. 48, no. 7, pp. 2930-2941, 2010.

[8] R. M. Narayanan, M. C. Shastry, P.-H. Chen, and M. Levi, "Through-the-wall detection of stationary human targets using Doppler radar," Progress in Electromagnetics Research B, no. 20, pp. 147-166, 2010.

[9] A. Marcello, B. Aniello, D. Michele, L. Angrisani, and R. S. lo Moriello, "A new measurement method based on music algorithm for through-the-wall detection of life signs," IEEE Transactions on Instrumentation and Measurement, vol. 62, no. 1, pp. 13-26, 2013. 
[10] C.-P. Lai, R. M. Narayanan, Q. Ruan, and A. Davydov, "HilbertHuang transform analysis of human activities using throughwall noise and noise-like radar," IET Radar, Sonar \& Navigation, vol. 2, no. 4, pp. 244-255, 2008.

[11] J. Li, Z. F. Zeng, J. G. Sun, and F. S. Liu, "Through-wall detectionof human being's movement by UWB radar," IEEE Geoscience and Remote Sensing Letters, vol. 9, no. 6, pp. 10791083, 2012.

[12] F. Ahmad and M. G. Amin, "Through-the-wall human motion indication using sparsity-driven change detection," IEEE Transactions on Geoscience and Remote Sensing, vol. 51, no. 2, pp. 881890, 2013.

[13] A. Buonanno, M. D'Urso, G. Prisco et al., "A new measurement method for through-the-wall detection and tracking of moving targets," Measurement, vol. 46, no. 6, pp. 1834-1848, 2013.

[14] Y. S. Yoon and M. G. Amin, "Spatial filtering for wall-clutter mitigation in through-the-wall radar imaging," IEEE Transactions on Geoscience and Remote Sensing, vol. 47, no. 9, pp. 3192-3208, 2009.

[15] B. J. Zhang and W. Wang, "Through-wall detection of human being with compressed UWB radar data," EURASIP Journal on Wireless Communications and Networking, vol. 51, no. 2, pp. 881890, 2013.

[16] F. Soldovieri, R. Solimene, and R. Pierri, "A simple strategy to detect changes in through the wall imaging," Progress in Electromagnetics Research M, vol. 7, no. 3, pp. 1-13, 2009.

[17] W. Zhang and A. Hoorfar, "Three-dimensional real-time through-the-wall radar imaging with diffraction tomographic algorithm," IEEE Transactions on Geoscience and Remote Sensing, vol. 51, no. 7, pp. 4155-4163, 2013.

[18] F. Ahmad, M. G. Amin, and G. Mandapati, "Autofocusing of through-the-wall radar imagery under unknown wall characteristics," IEEE Transactions on Image Processing, vol. 16, no. 7, pp. 1785-1795, 2007.

[19] M. M. Riaz and A. Ghafoor, "Through-wall image enhancement based on singular value decomposition," International Journal of Antennas and Propagation, vol. 2012, Article ID 961829, 20 pages, 2012.

[20] T. Jin, B. Chen, and Z. Zhou, "Image-domain estimation of wall parameters for autofocusing of through-the-wall SAR imagery," IEEE Transactions on Geoscience and Remote Sensing, vol. 51, no. 3, pp. 1836-1843, 2013

[21] E. M. Lavely, Y. Zhang, E. H. Hill III, Y.-S. Lai, P. Weichman, and A. Chapman, "Theoretical and experimental study of throughwall microwave tomography inverse problems," Journal of the Franklin Institute, vol. 345, no. 6, pp. 592-617, 2008.

[22] P. Setlur, M. Amin, and F. Ahmad, "Multipath model and exploitation in through-the-wall and urban radar sensing," IEEE Transactions on Geoscience and Remote Sensing, vol. 49, no. 10, pp. 4021-4034, 2011

[23] J. Wang, P. F. Wang, and Y. H. Li, "A multipath suppression technique for through radar," in Proceedings of the IEEE International Conference on Ultra-Wideband (ICUWB '13), pp. 1-6, Sydney, Australia, 2013.

[24] S. E. Borek, "An overview of through the wall surveillance for homeland security," in Proceedings of the 34th Applied Imagery and Pattern Recognition Workshop, pp. 42-47, Washington, DC, USA, October 2005.

[25] A. Hunt, "Random array through the wall imaging sensor," Tech. Rep. AFRL-IF-RS-TR-2006-122, The Air Force Research Laboratory, Rome, NY, USA, 2006.
[26] C. Debes, Advances in Detection and Classification for Throughthe-Wall Radar Imaging, Darmstadt University of Technology, Darmstadt, Germany, 2010.

[27] A. Beeri and R. Daisy, "High-resolution through-wall imaging," in Sensors, and Command, Control, Communications, and Intelligence (C3I) Technologies for Homeland Security and Homeland Defense V, vol. 6201 of Proceedings of SPIE, Orlando, Fla, USA, 2006.

[28] T. Dogaru and C. Le, "SAR images of rooms and buildings based on FDTD computer models," IEEE Transactions on Geoscience and Remote Sensing, vol. 47, no. 5, pp. 1388-1401, 2009.

[29] S. Xin, Z. Zhimin, L. Biying, and J. Tian, "A fast direct wave suppression method by weighting in through the wall imaging," in Proceedings of the 2nd International Conference on Intelligent Systems Design and Engineering Applications (ISDEA '12), pp. 971-974, Sanya, China, January 2012.

[30] M. Dehmollaian and K. Sarabandi, "Refocusing through building walls using synthetic aperture radar," IEEE Transactions on Geoscience and Remote Sensing, vol. 46, no. 6, pp. 1589-1599, 2008.

[31] B. Lu, Q. Song, Z. Zhou, and H. Wang, "A SFCW radar for through wall imaging and motion detection," in Proceedings of the 14th European Microwave Week (EuMW '11), pp. 1-5, Manchester, UK, October 2011. 

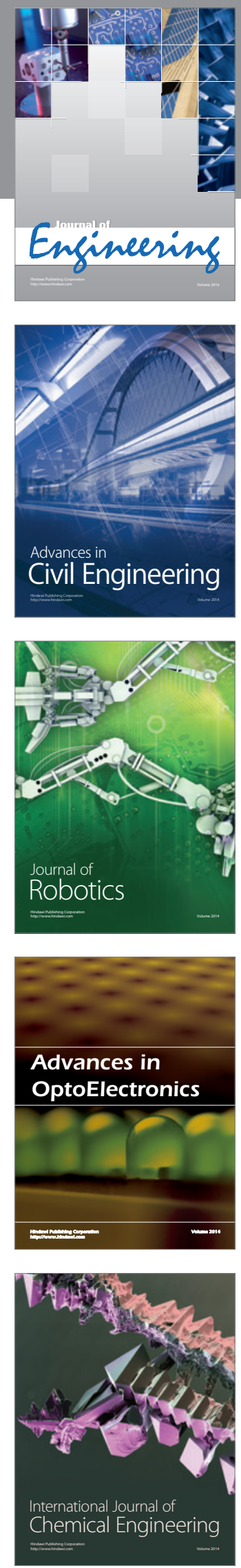

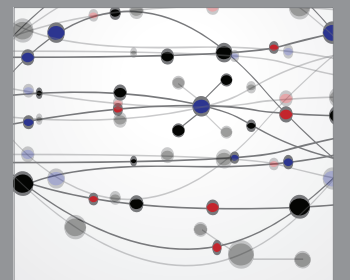

The Scientific World Journal
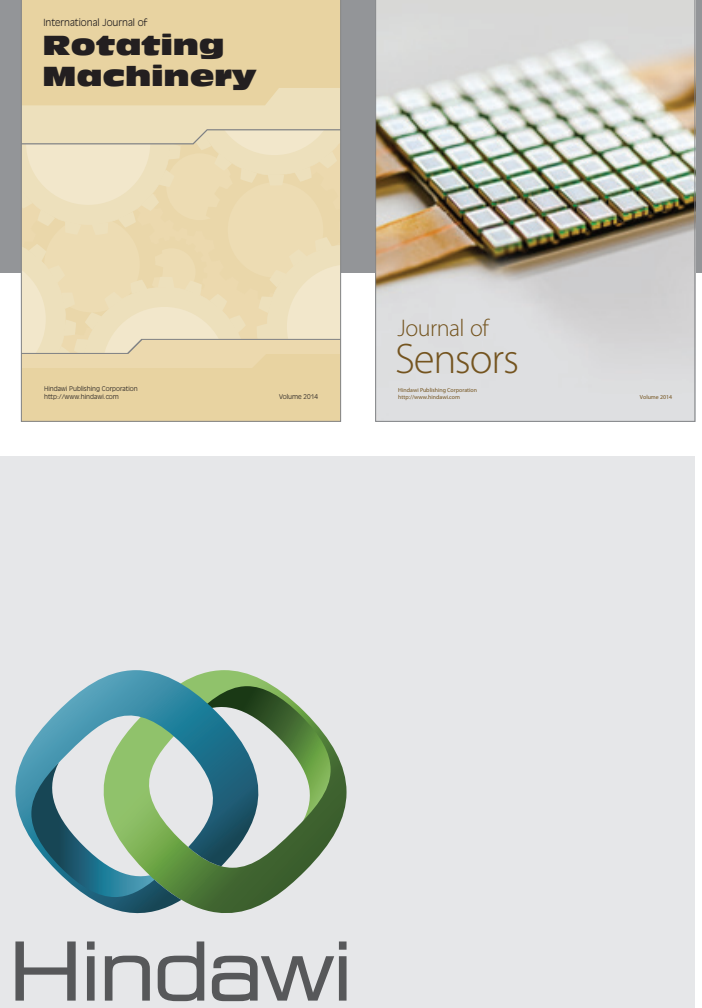

Submit your manuscripts at http://www.hindawi.com
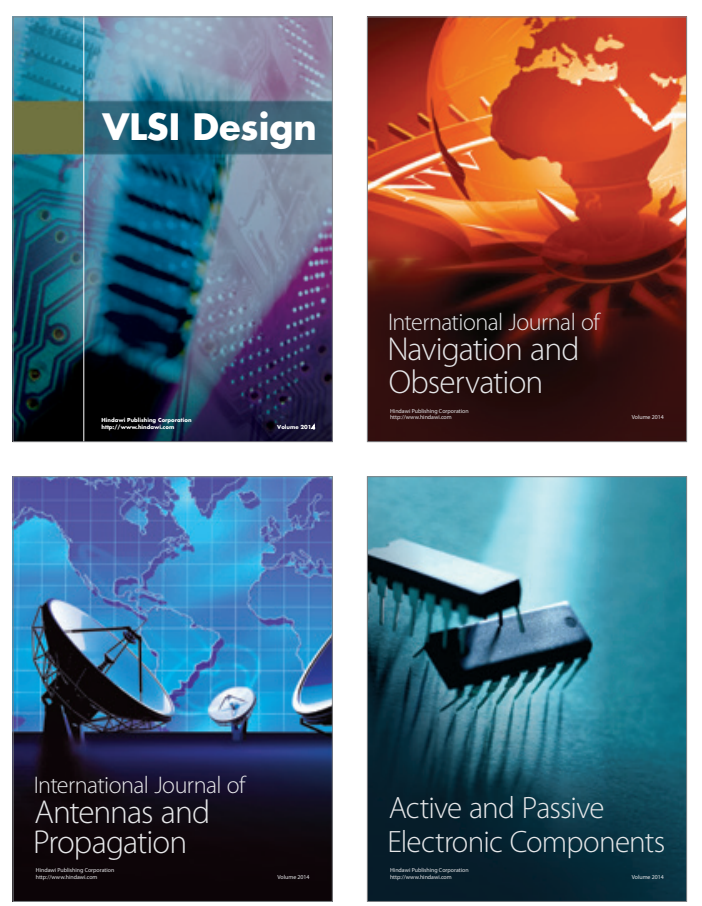
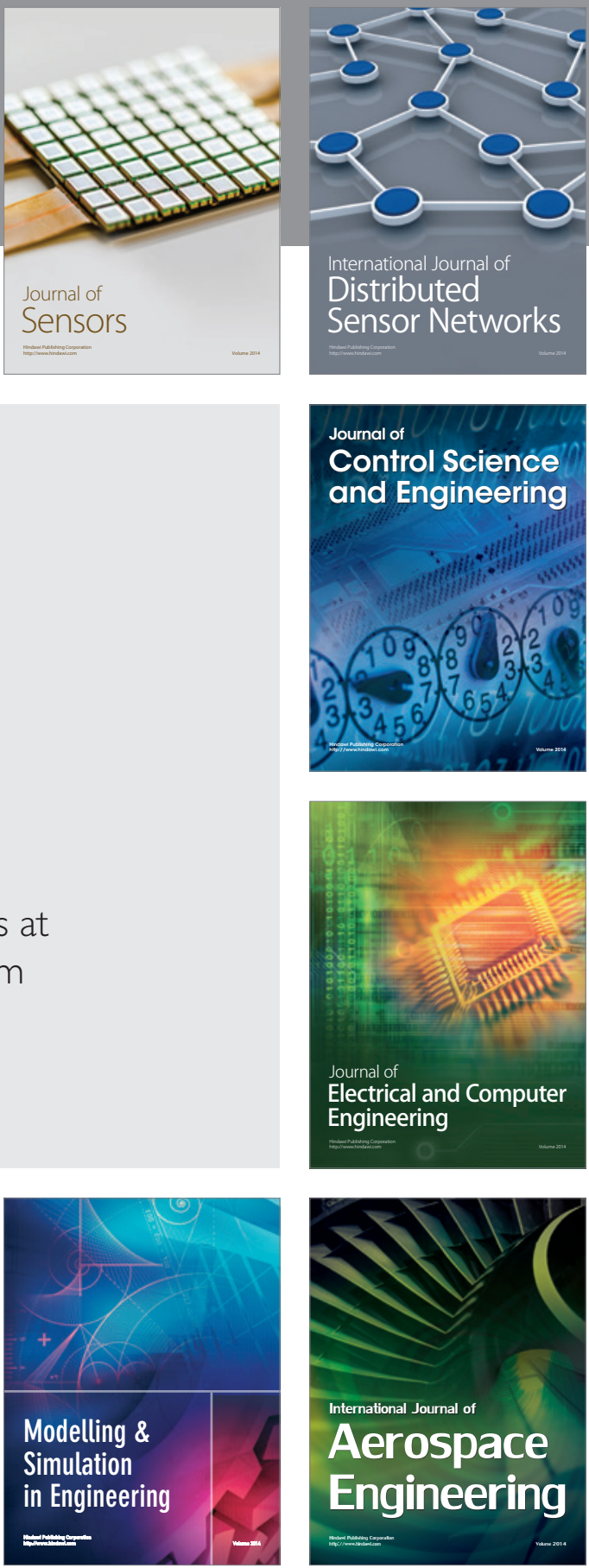

Journal of

Control Science

and Engineering
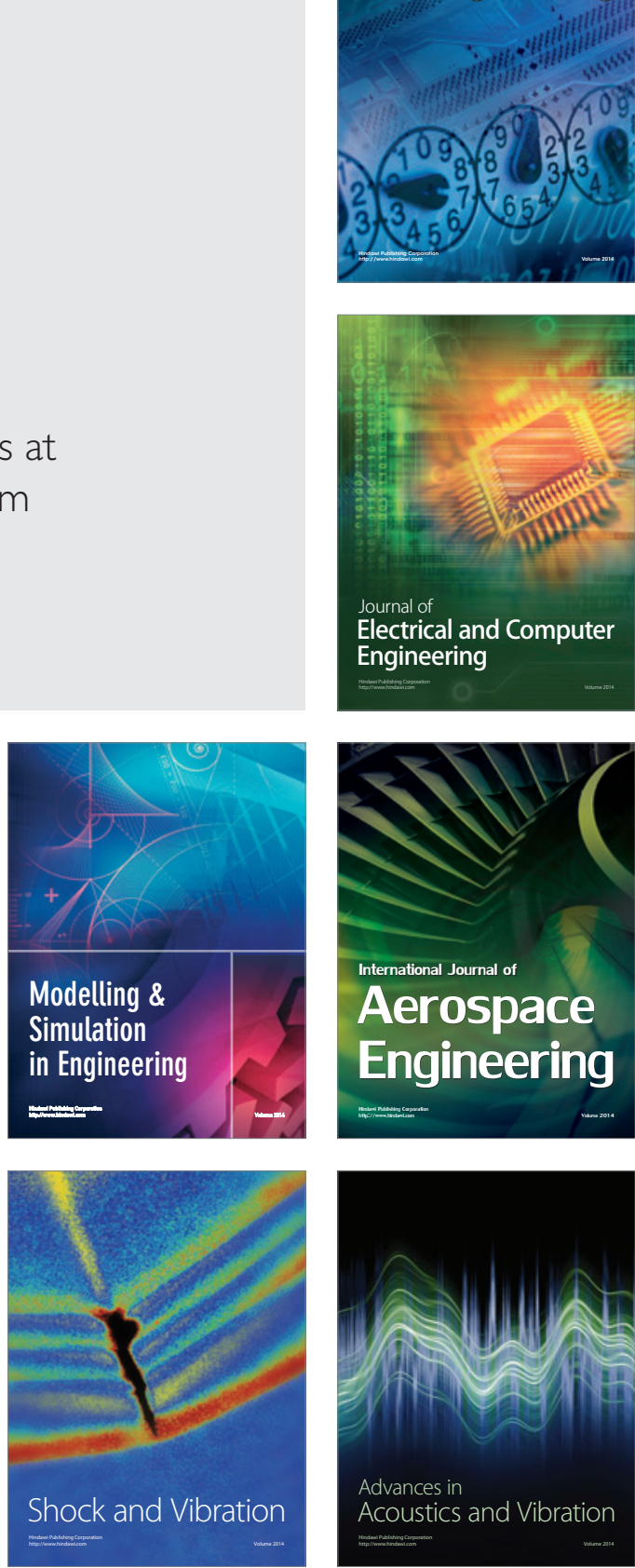